ARTICLE

DOI: $10.1038 / s 41467-018-05100-z$

\title{
Microglia permit climbing fiber elimination by promoting GABAergic inhibition in the developing cerebellum
}

\author{
Hisako Nakayama ${ }^{1,2}$, Manabu Abe ${ }^{3,4}$, Chie Morimoto ${ }^{1,5}$, Tadatsune lida6, Shigeo Okabe (i) ${ }^{6}$, \\ Kenji Sakimura ${ }^{3,4}$ \& Kouichi Hashimoto (i) ${ }^{1}$
}

Circuit refinement during postnatal development is finely regulated by neuron-neuron interactions. Recent studies suggest participation of microglia in this process but it is unclear how microglia cooperatively act with neuronal mechanisms. To examine roles of microglia, we ablate microglia by microglia-selective deletion of colony-stimulating factor 1 receptor (Csf1r) by crossing floxed-Csf1r and Iba7-iCre mice (Csf1r-cKO). In Csf1r-cKO mice, refinement of climbing fiber (CF) to Purkinje cell (PC) innervation after postnatal day 10 (P10)-P12 is severely impaired. However, there is no clear morphological evidence suggesting massive engulfment of CFs by microglia. In Csflr-cKO mice, inhibitory synaptic transmission is impaired and CF elimination is restored by diazepam, which suggests that impairment of $\mathrm{CF}$ elimination is caused by a defect of GABAergic inhibition on PCs, a prerequisite for CF elimination. These results indicate that microglia primarily promote GABAergic inhibition and secondarily facilitate the mechanism for CF elimination inherent in PCs.

\footnotetext{
${ }^{1}$ Department of Neurophysiology, Graduate School of Biomedical and Health Sciences, Hiroshima University, 1-2-3 Kasumi, Minami-ku, Hiroshima $734-8551$ Japan. ${ }^{2}$ Department of Physiology, School of Medicine, Tokyo Women's Medical University, 8-1 Kawada-cho, Sinjuku-ku, Tokyo 162-8666, Japan.

${ }^{3}$ Department of Cellular Neurobiology, Brain Research Institute, Niigata University, 1-757 Asahimachi-dori, Chuo-ku, Niigata 951-8585, Japan. ${ }^{4}$ Department of Animal Model Development, Brain Research Institute, Niigata University, 1-757 Asahimachi-dori, Chuo-ku, Niigata 951-8585, Japan. ${ }^{5}$ Department of Psychosocial Rehabilitation Graduate School of Biomedical \& Health Sciences, Hiroshima University, 1-2-3 Kasumi, Minami-ku, Hiroshima 734-8551, Japan. ${ }^{6}$ Department of Cellular Neurobiology, Graduate School of Medicine, University of Tokyo, 7-3-1 Hongo, Bunkyo-ku, Tokyo 113-0033, Japan. These authors contributed equally: Hisako Nakayama, Manabu Abe. Correspondence and requests for materials should be addressed to K.H. (email: hashik@hiroshima-u.ac.jp)
} 
$\mathrm{M}$ ost microglia derive from primitive myeloid progenitors that originate in the extraembryonic yolk sac and penetrate into the brain from embryonic day 8.5 in mice ${ }^{1}$. Microglia dynamically change their distribution and morphology during development ${ }^{2-8}$. Ramified microglia evenly distribute across the entire adult brain irrespective of whether it is gray or white matter (WM), but distribution of immature microglia with ameboid morphology is biased to the medulla and ventricular/subventricular zones around the time of birth ${ }^{2-5,7-9}$. Because microglia in the vicinity of targets act by direct contact, a

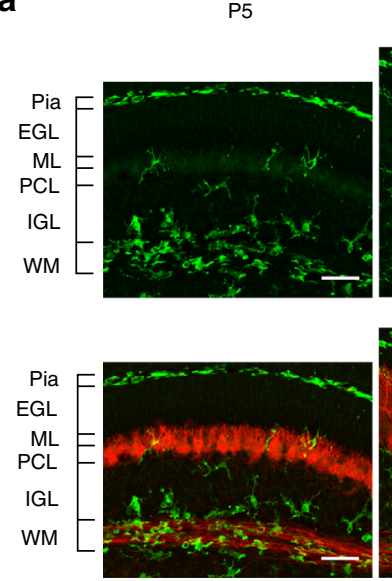

b
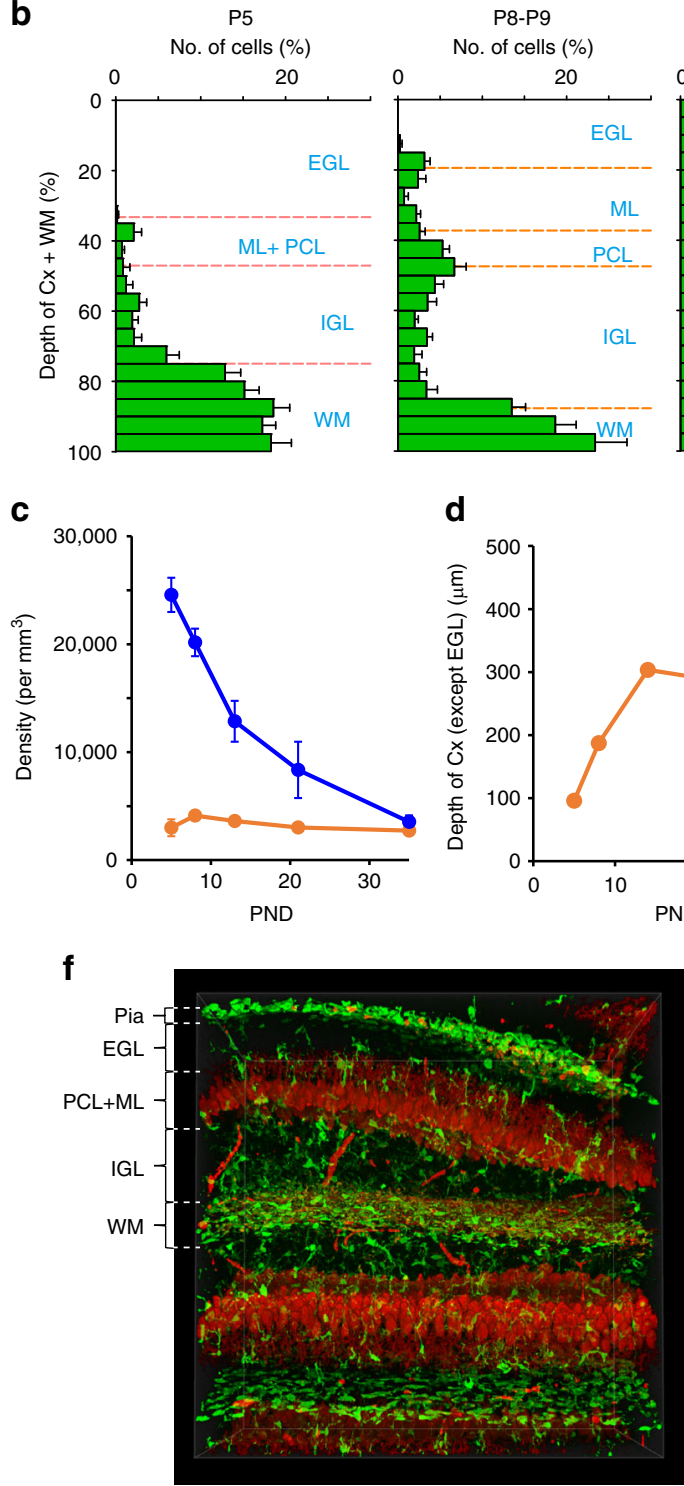

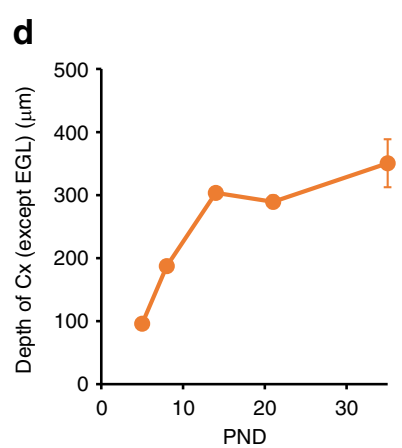

P13-P14
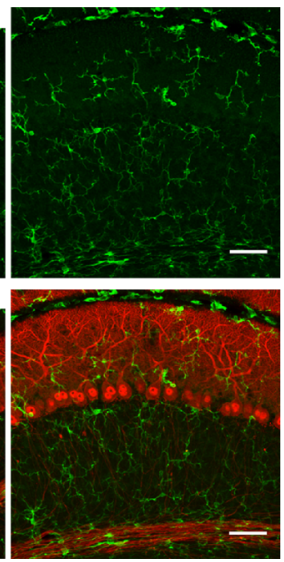

P8-P9

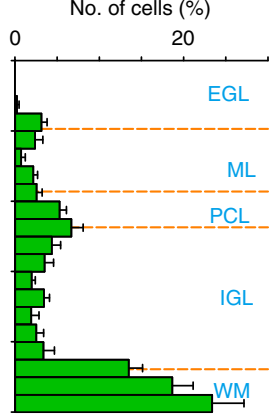

P13-P14
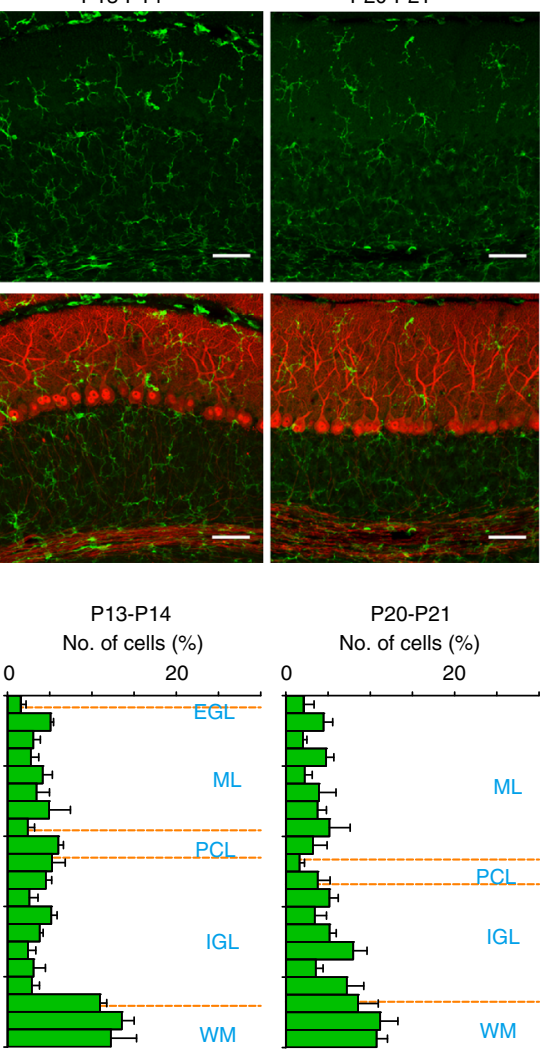

P20-P21 No. of cells $(\%)$
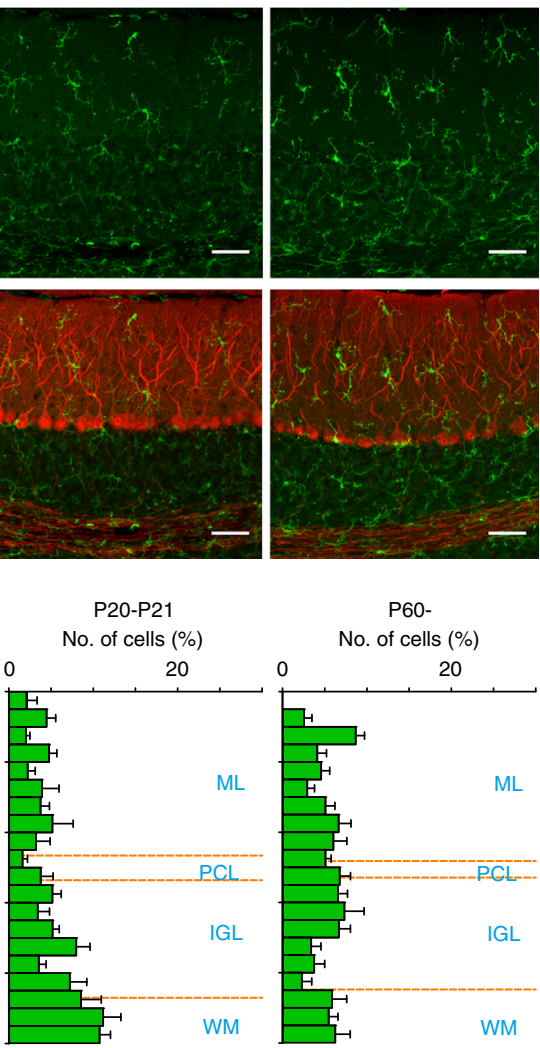

e
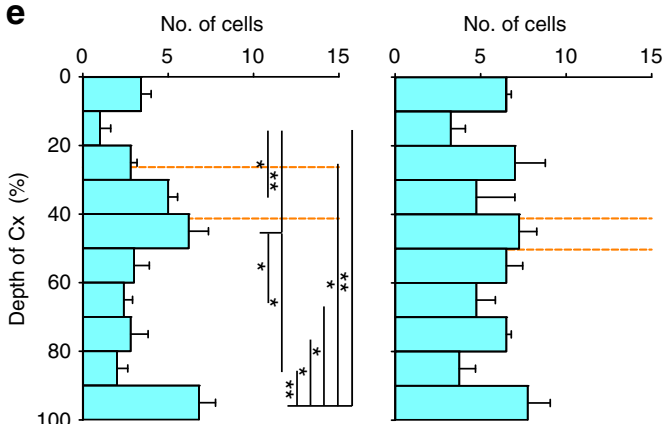

o. of cells (\%)

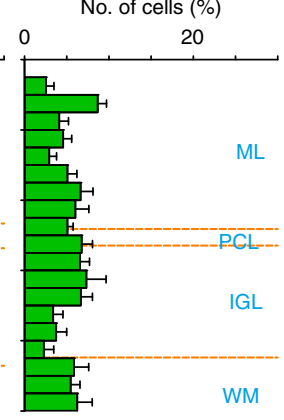

\section{g}

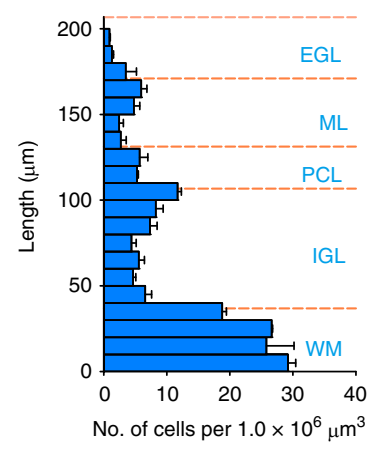


release of bioactive molecules or phagocytosis ${ }^{10,11}$, their influential range is relatively restricted. Therefore, the functional roles of microglia likely change with the progress of their translocation in the developing brain ${ }^{8,12}$, and it is important to analyze the functional roles of microglia in association with changes in their spatial distribution.

Microglia play indispensable roles in shaping neuronal circuits in various ways in the developing central nervous system. They regulate the number of neurons by promoting apoptotic cell death ${ }^{13}$ and removal of neural precursors by phagocytosis ${ }^{5}$. In addition, microglia remodel synaptic connections. Neuronal circuits are initially redundant at birth but are gradually refined during postnatal development. Many studies have proposed that these developmental processes are regulated by an interplay between neurons ${ }^{14,15}$. However, recent studies have disclosed that microglia promote spine and excitatory synapse formation ${ }^{16-18}$ and elimination of redundant excitatory synapses ${ }^{18-23}$ during postnatal circuit refinement. Some synaptic connections are thought to be removed by engulfment by microglia ${ }^{19-23}$, but it is largely unclear how microglia participate in a coordinated manner in postnatal circuit refinement regulated by neuronal mechanisms.

The cerebellar cortex is an appropriate model system to study the mechanisms for constructing functional neuronal connections. The majority of cerebellar Purkinje cells (PCs) receive inputs from only one climbing fiber (CF) in the adult but are innervated by multiple CFs at birth. One $\mathrm{CF}$ is selectively strengthened on the PC soma relative to the others by postnatal day $7(\mathrm{P} 7)^{15}$. The single winner $\mathrm{CF}$ then undergoes translocation to the PC's dendrites ${ }^{24}$ and extensive parallel fiber $(\mathrm{PF})$ synapse formation proceeds $\mathrm{s}^{25}$. In parallel, redundant CFs are eliminated ${ }^{15,26}$ and PC somata are instead innervated by inhibitory basket cells ${ }^{27}$. The construction of cerebellar circuits is thought to be finely regulated by neuron-neuron interactions ${ }^{28-30}$. Although factors necessary for such neuron-neuron interactions have been extensively studied $^{15,28-30}$, the roles of non-neuronal cells, such as glial cells, are largely unclear.

In the present study, we mainly focused on the postnatal development of mouse CF-PC synapses to elucidate the roles of microglia in neuronal circuit development. For this purpose, microglia were ablated from the developing cerebellum by cerebellar injection of clodronate liposomes or by microglia-selective deletion of the colony-stimulating factor 1 receptor (Csflr) gene (Csflr-cKO). We found that CF synapse elimination is severely impaired in these mice. Importantly, inhibitory synaptic transmission was attenuated in Csf1r-cKO mice and CF elimination was restored by administration of a $\mathrm{GABA}_{\mathrm{A}}$ receptor sensitizer, diazepam. These results suggest that microglia primarily enhance GABAergic synaptic transmission to PCs during postnatal development, thereby indirectly promoting the process of CF synapse elimination that is controlled by the interplay between inhibitory neurons and PCs.

\section{Results}

Biased distribution of microglia beneath the Purkinje cell layer (PCL) at P8-P9. We examined the developmental changes in microglial distribution in the cerebellum. At P5, the density of microglia was much higher in the WM than in the gray matter (internal granule cell layer: IGL, Purkinje cell layer: PCL, and molecular layer: ML) (Fig. 1a, b), consistent with a previous report $^{7}$. The majority of the Iba1-positive microglia in the WM at P5 were ameboid, with large somata that were devoid of long processes (Supplementary Fig. 1). After P8-P9, the localization of microglia to the WM gradually decreased in parallel with the growth of the cerebellar cortex ${ }^{31}$ (Fig. 1a-d), to finally become almost evenly distributed in the cerebellum at P60 (Fig. 1a, b). The morphology of the Iba1-positive microglia in the gray matter also changed after P8-P9. Their processes became thinner and more branched, and their somata became smaller as development proceeded (Supplementary Fig. 1). As previously suggested in quail and rat cerebellum ${ }^{4,7}$, invasion of microglia into the developing cerebellar gray matter mainly occurred from the WM, because the density of microglia was higher on the WM side of the IGL and the external granule cell layer (EGL) was almost devoid of microglial somata (Fig. 1a, b).

Notably, we found that the density of microglial somata was significantly higher around the area from the PCL to the IGL that peaked beneath the PCL at P8-P9 (Fig. 1e, left). This biased distribution was not observed at P13-P14 (Fig. 1e, right). We confirmed this biased distribution using higher-volume specimens (Fig. 1f). Wild-type mouse brains were optically cleared by $\mathrm{SeeDB} 2^{32}$, and the distribution of microglia was examined in a volume of $200 \times 500 \times 500 \mu^{3}$ for each animal $(n=3$ mice $)$. At P8-P9, the density of microglial somata had a large peak beneath the PCL and a smaller one at the edge between the ML and the EGL (Fig. 1g). Taken together, this transient and biased distribution of microglia raises the possibility that they may affect postnatal development of neuronal circuits in the cerebellar cortex at P8-P9.

Pharmacological ablation of microglia impairs CF elimination. The number of CFs innervating a single PC gradually increases during the first postnatal week, but starts to decrease after P7 in the mouse cerebellum ${ }^{33}$. The transient microglial accumulation we observed beneath the PCL is almost coincident with the start of CF elimination, which suggests a contribution by microglia to CF elimination. To test this possibility, we ablated microglia by infusion of liposomal clodronate into cerebellar lobules VI/VII or VIII at P6 or P7, around the start of CF elimination in wild-type mice. Liposomal clodronate is engulfed by phagocytic cells and induces cell death ${ }^{14}$. Immunohistochemical staining revealed that microglia locally disappeared from the cerebellar cortex around the injection site by P8 (Fig. 2a-c). Severe morphological alterations of calbindin-labeled PCs were not apparent (Fig. 2a, b). Importantly, injection of empty liposomes did not deplete

Fig. 1 Postnatal changes in microglial distribution in the cerebellum. a Immunofluorescent labeling for lba1 (green) and calbindin staining (red) in lobules IV-V in the vermis of mouse cerebellum at P5, P8, P14, P21, and P60. EGL external granule cell layer, ML molecular layer, PCL Purkinje cell layer, IGL internal granule cell layer, WM white matter. Scale bars, $50 \mu \mathrm{m}$. b Frequency distribution histograms for the relative depth of microglia at P5 ( $n=5$ mice), P8-P9 $(n=5), \mathrm{P} 13-\mathrm{P} 14(n=4), \mathrm{P} 20-\mathrm{P} 21(n=4)$, and P60 $(n=6)$. The depth of microglia was normalized to the total length of the gray matter and the white matter where each microglial cell was sampled. c Postnatal changes in densities of microglia in the WM (blue) and the gray matter (orange). Numbers of mice at individual postnatal days are same as in (b). d Postnatal changes in the total length of the ML, PCL, IGL, and WM. e Frequency distribution histograms of the relative depth of microglia in the gray matter except the EGL (the ML, the PCL, and the IGL) at P8-P9 (left, $n=5$ mice) and P13-P14 (right, $n=4$ ). Note that microglia tend to aggregate beneath the part of the PCL (the area between orange broken lines) and the edge beside the WM at P8-P9; ${ }^{\star} p<0.05,{ }^{\star \star} p<0.01$. $\mathrm{f}$ Volume rendered image $(508 \times 508 \mu \mathrm{m}, 450 \mu \mathrm{m}$ depth) of the mouse cerebellum at P8 with immunofluorescent labeling of Iba1 (green) and calbindin (red). $\mathbf{g}$ Density of microglia across the layers ( $n=3$ mice). Data are presented as mean \pm SEM 
Clodronate-liposime at P6, Fix at P8
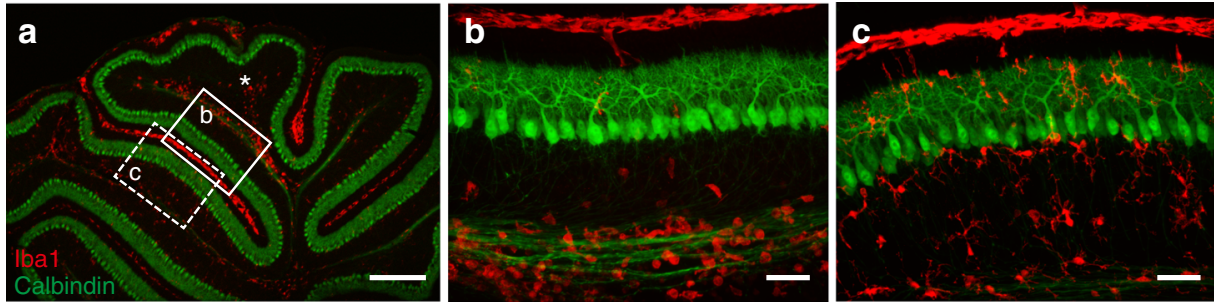

Control liposome at P6, Fix at P8
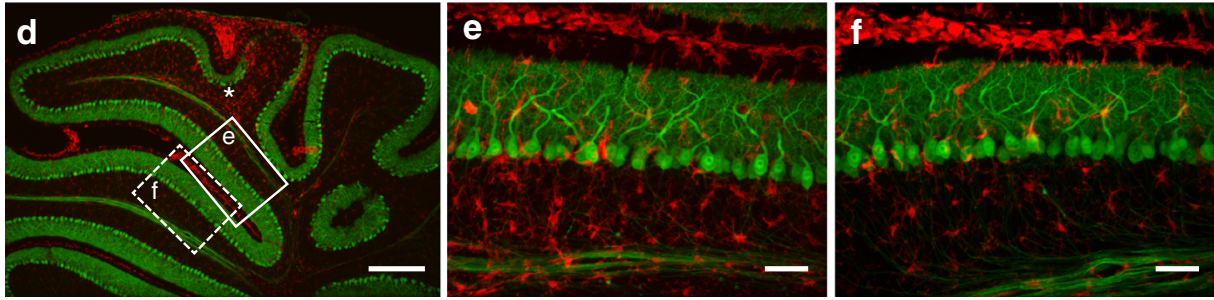

g Injection at P6, electrophysiology at P21-P31

h Injection at P11, electrophysiology at P21-P31
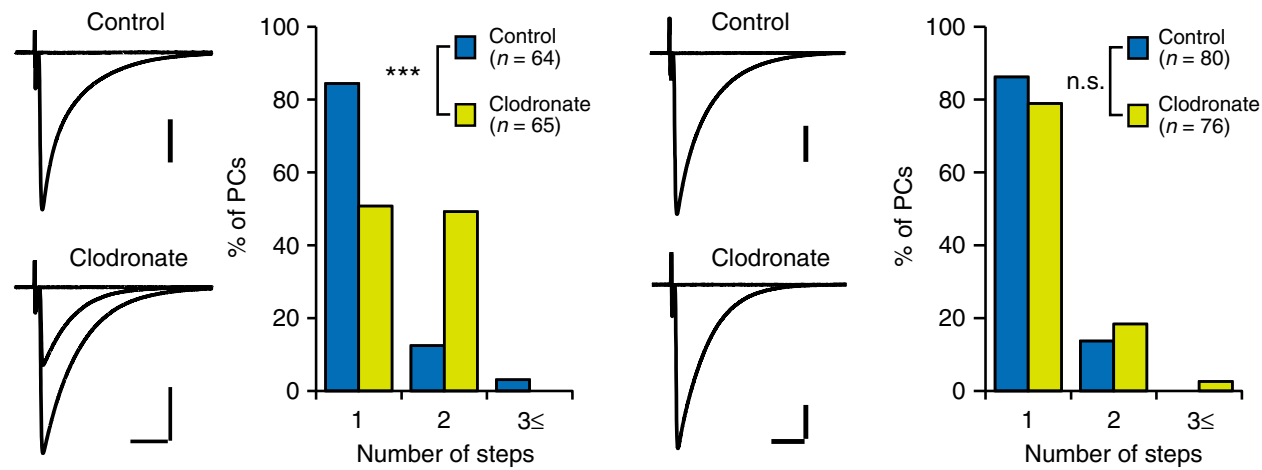

Fig. 2 Effects of pharmacological deletion of microglia on CF synapse elimination. a-f Double immunolabeling for Iba1 (red) and calbindin (green) in liposomal clodronate (a-c) or control liposome (d-f) injected mice at P8. Asterisks in a and $\mathbf{d}$ indicate the injection sites of the liposomes in lobules VI-VII in the vermis. Regions surrounded by solid and dotted lines are magnified in $\mathbf{b}$ and $\mathbf{e}$, and $\mathbf{c}$ and $\mathbf{f}$, respectively. Iba1-labeled microglial cell numbers are significantly reduced in the clodronate-injected lobules VI-VII (b), but not in the neighboring lobules IV-V (c). $\mathbf{g}$, $\mathbf{h}$ (left) Representative traces of CF-EPSCs recorded in mice treated with control (upper) or clodronate (lower) liposomes at P6 (g) or P11 (h). Electrophysiological recordings were conducted from PCs in the liposome-injected lobules at P21-P31. The holding potential (Vh) is $-10 \mathrm{mV}$. Scale bars, $1 \mathrm{nA}(\mathbf{g}), 0.5 \mathrm{nA}(\mathbf{h})$ and $10 \mathrm{~ms}$. (right) Frequency distribution histograms showing the number of discrete CF-EPSC steps. Data were sampled from five control and five clodronate-treated mice in each of $\mathbf{g}$ and $\mathbf{h}$. CF synapse elimination was significantly impaired in mice treated with clodronate at P6 (g, $p<0.001$, Mann-Whitney $U$ test), but not at P11 (h, $p=$ 0.290). Scale bars, $300 \mu \mathrm{m}(\mathbf{a}, \mathbf{d})$ and $50 \mu \mathrm{m}(\mathbf{b}, \mathbf{c}, \mathbf{e}, \mathbf{f}) .{ }^{\star \star \star} p<0.001 ;$ n.s., $p>0.05$

microglia (Fig. 2d-f). These results confirmed the local ablation of microglia by injection of clodronate liposomes.

Acute cerebellar slices were prepared from control or clodronate liposome-injected mice at P21-P31, when circuit refinement in the cerebellar cortex was almost completed. CFmediated excitatory postsynaptic currents (CF-EPSCs) were recorded from PCs in liposome-injected lobules (Fig. 2g). In nearly $85 \%$ of PCs in control liposome-injected mice, CF-EPSCs were elicited in an all-or-nothing manner when the stimulus intensity was gradually increased (Fig. 2g, control). Conversely, in nearly $50 \%$ of PCs in the clodronate liposome-treated cerebellum, CF-EPSCs were elicited in a stepwise manner with increases in the stimulus intensity (Fig. 2g, clodronate). Because the number of CF-EPSC steps represents the number of CFs innervating a given PC, these results suggest that ablation of cerebellar microglia from P6-P8 severely impairs CF elimination. The kinetics of CF-EPSCs in mono-innervated PCs were not different between clodronate and control liposome-treated mice (Supplementary Table 1).
To examine the critical period for the clodronate-induced impairment of CF elimination, liposomal clodronate was injected at P11. Immunostaining confirmed that Iba1-positive microglia were successfully reduced at P13 (Supplementary Fig. 2) but CF elimination was not impaired in these mice (Fig. 2h). These results suggest that the critical period for microglia-dependent CF elimination was already closed by P11-P13.

We next examined the effect of pharmacological activation of microglia on CF synapse elimination. Lipopolysaccharide (LPS, 0.3 or $1.5 \mu \mathrm{g} \mathrm{g}^{-1}$ body weight) or PBS was injected intraperitoneally at P7-P11 or P12-P16, and CF-EPSC steps were analyzed electrophysiologically after P21 (Supplementary Fig. 3). LPS administration at both postnatal periods effectively changed the morphology of the microglia (Supplementary Fig. 3a-f). However, there was no significant difference in the number of CF-EPSC steps in PCs of PBS-, $0.3 \mu \mathrm{gg}^{-1}$ LPS-, or $1.5 \mu \mathrm{g} \mathrm{g}^{-1}$ LPS-treated mice for either administration period (Supplementary Fig. 3g, h). These results suggest that LPS-induced activation of microglia does not affect elimination of surplus CFs. Basal 
a

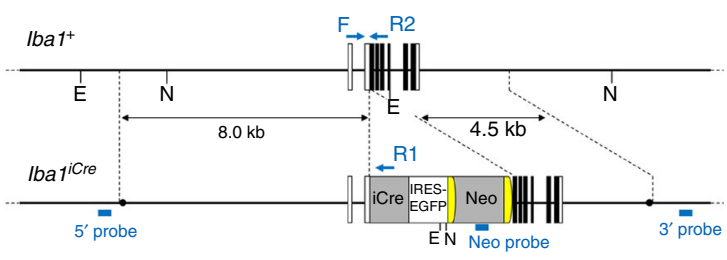

b

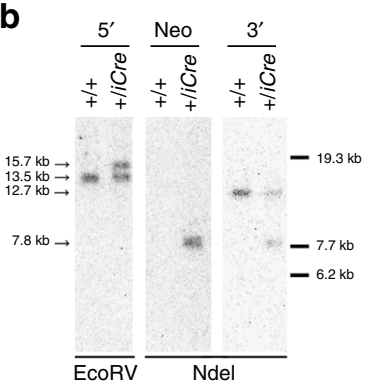

C
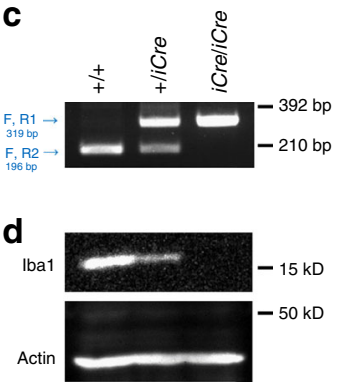
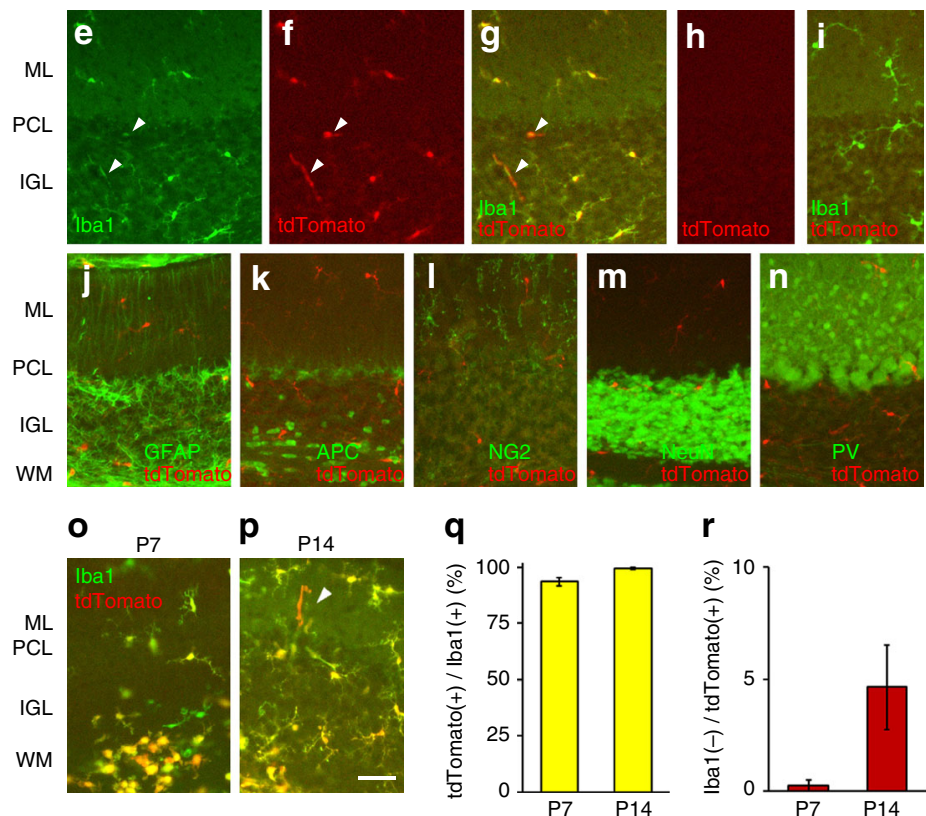

Fig. 3 Generation of the Iba1-iCre mouse line. a Schema of Iba1 genomic DNA and the targeted genome. Open and filled boxes indicate noncoding and coding exons, respectively. Filled circles in the $\mathrm{Iba} 7^{i C r e}$ allele delineate the $5^{\prime}$ and $3^{\prime}$ termini of the targeting vector. The vector was constructed to insert an improved Cre recombinase gene (iCre), followed by an internal ribosome entry site (IRES) and a FLAG-tagged EGFP, in frame into the translational initiation site of the Iba1 gene. Blue arrows show primer positions for PCR genotyping. Blue bars indicate probes for Southern blot analysis. Two frt sequences (semicircles) are attached to remove the neomycin resistance gene (Neo). E, EcoRV; N, Ndel. b Southern blot analysis for genomic DNAs from wild-type $(+/+)$ and targeted (+/iCre) ES cells. Left, EcoRV-digested genomic DNA hybridized with 5' probe; middle and right, Ndel-digested DNA hybridized with Neo or 3' probe, respectively. c Genomic PCR genotyping of Iba1 ${ }^{+/ i C r e}$ intercrosses using primers F, R1 and R2. d Immunoblots of crude fractions from whole mouse brains with anti-Iba1 and actin antibodies. e-i Cre recombinase activity induced by Iba1-iCre in CAG-floxed STOP tdTomato reporter mice. No EGFP fluorescence was detected in the Iba1-iCre cerebellum. Immunostaining for lba1 (green) and tdTomato fluorescence (red) in lobules IV-V in the

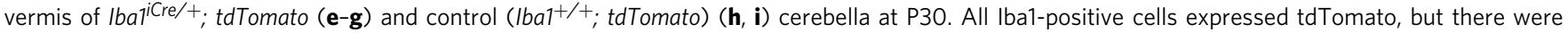
several tdTomato-labeled processes with very weak or no lba1 immunoreactivity (arrowheads in e-p). j-n Immunostaining for cell markers (green) in Iba7 $\mathrm{I}^{\mathrm{Cre} /+}$; tdTomato mice at P30; tdTomato did not overlap with markers for astrocyte (GFAP, j), oligodendrocyte (APC, k), NG2 chondroitin sulfate proteoglycan (I), neurons (NeuN, m) or inhibitory neurons (parvalbumin, $\mathbf{n}$ ). o-r Localization of Iba1 and tdTomato signals at P7 (o) or P14 (p). $\mathbf{q}, \mathbf{r}$ Fractions of tdTomato-labeled cells relative to lba1-positive cells $(\mathbf{q})$ or lba1-negative cells relative to tdTomato-labeled cells $(\mathbf{r})$ ( $n=310$ cells from two mice at P7, $n=191$ cells from two mice at P14). A scale bar, $50 \mu \mathrm{m}$ (e-p). Error bars, SEM

activity of microglia may be sufficient to promote CF elimination in the developing cerebellum.

Conditional deletion of Csf1r in microglia. Clodronate liposome administration successfully deleted microglia from the cerebellum. However, we cannot completely rule out the possibility that undetectable damage to other off-target cells with phagocytic activity, such as astrocytes, caused impairment of CF elimination in those experiments. Therefore, we next more selectively ablated microglia using cell-selective genetic manipulation. Previous reports demonstrated that CSF1R is a critical molecule for differentiation and survival of microglia in the brain ${ }^{34,35}$. To ablate microglia from developing cerebellum, we conditionally deleted Csf1r from microglia by crossing floxed Csf1r mice and Iba1-iCre knock-in mice to generate Csf1r(lox/lox); Ibal(iCre/iCre) mice, hereafter referred as Csf1r-cKO mice. Littermates without Cre recombinase (Csf1r(lox/lox); Iba1(wt/wt)) were used as controls.

First, we generated the Ibal-iCre mouse and examined the expression pattern of an iCre in the developing cerebellum (Fig. 3). Distribution of iCre-expressing cells was analyzed using a reporter mouse line (Ibal(iCre/+); CAG-floxed STOP tdTomato), which was produced by intercrossing the Iba1-iCre line with the Cre-inducible tdTomato reporter mouse line (CAG-floxed STOP tdTomato, Supplementary Fig. 4). TdTomato was selectively expressed in microglia (Fig. $3 \mathrm{e}-\mathrm{i}$ ) but not in neurons and other types of glial cells (astrocytes, oligodendrocytes, or NG2-positive cells) (Fig. 3j-n). TdTomato was already expressed at P7 in about 95\% of Iba1-positive cells (Fig. 3o, q). At P14, there was a minor population of Iba1-negative but td Tomato-positive cells that associated with some blood vessels (Fig. $3 \mathrm{p}, \mathrm{r}$ ) in the cerebellar cortex.

The Csflr-cKO mice grew to adulthood and were fertile. Their brain structures, the midline crossing of callosal axons and cortical thickness were largely normal, but ventricular size was enlarged at P35 (Supplementary Fig. 5). Overall foliation of the cerebellum was normal in the Csflr-cKO mice (Supplementary Fig. 6a, b, e, f). In addition, the thickness of the granule cell layer in Csf1r-cKO mice was not significantly different from that in control mice (Supplementary Fig. 6c, g, r). The thickness of the $\mathrm{ML}$ was significantly increased in Csf1r-cKO mice, but the absolute difference was slight (Supplementary Fig. 6b, f, q). The density of somata of PCs, interneurons in the ML, or Golgi cells in the IGL was also normal (Supplementary Fig. 6d, h, i, m, s-u). These results indicate that the overall structure of the cerebellum is normal in Csflr-cKO mice.

Next, we assessed reduction of microglia in the cerebellum with an antibody for CX3CR1, because Iba1 was completely deleted in Csflr-cKO mice. In control mice, Ibal-positive microglia in the developing cerebellum colocalized with the CX3CR1 signals (Fig. $4 \mathrm{a}-\mathrm{i}$ ). However, the density of CX3CR1-positive microglia was severely reduced at P7 in Csflr-cKO mice (Fig. 4j, o). Their 


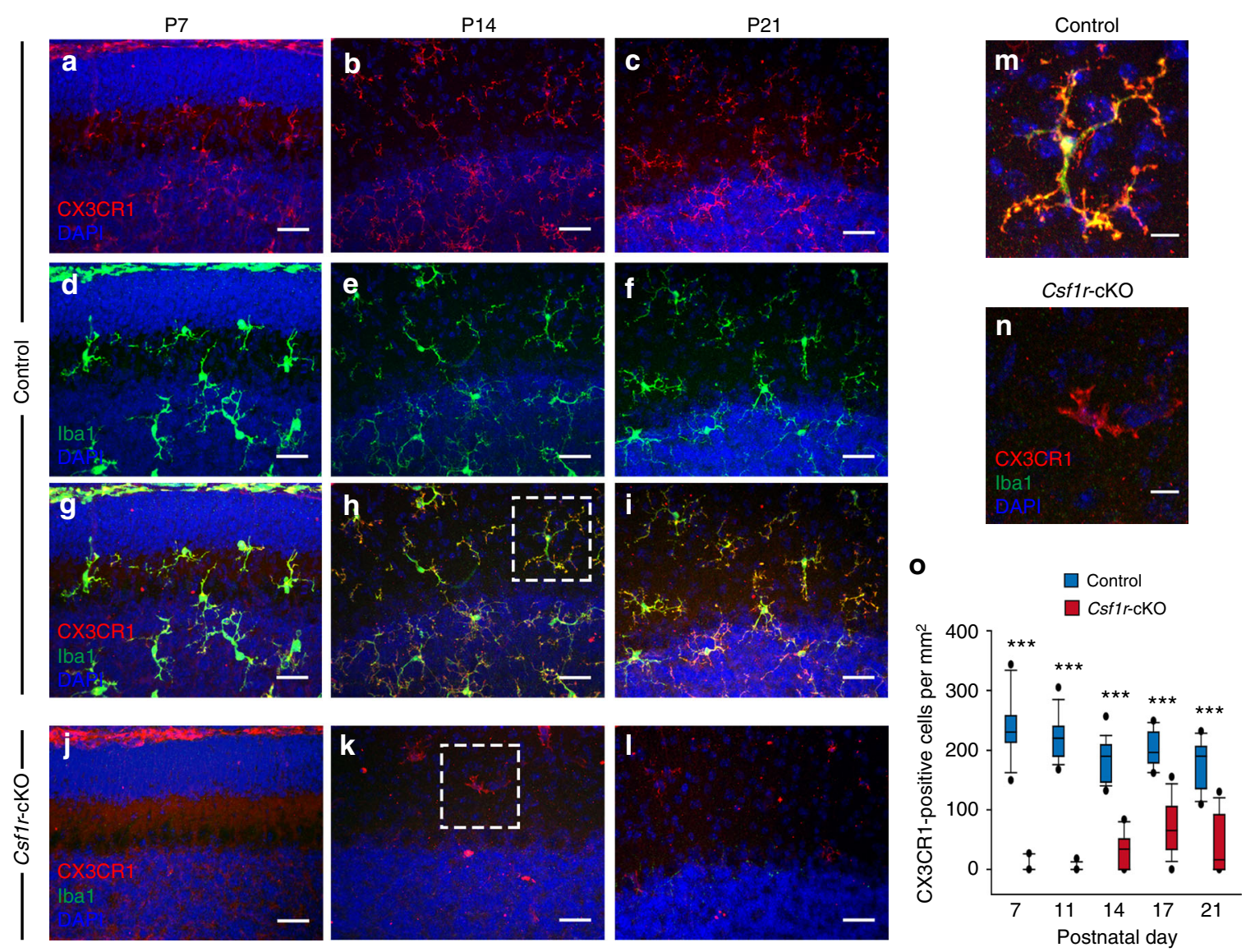

Fig. 4 Microglia-selective deletion of Csf1r results in significant loss of microglia in the developing cerebellum. a-I Immunostaining for CX3CR1 (red), Iba1 (green), and DAPI (blue) in control mice (a-i) and Csf1r-cKO mice (j-I) at P7 (left), P14 (middle), and P21 (right). Images are maximum z-projections of slices with thicknesses of $50 \mu \mathrm{m}$ taken from lobules VI-V in the vermis. In Csf1r-cKO mice, CX3CR1-positive cells were almost absent at P7 (j), and sparse at P14 (k) and P21 (I). $\mathbf{m}, \mathbf{n}$ Enlargements of the CX3CR1-positive cells surrounded by dashed squares in $\mathbf{h}$ and $\mathbf{k}$, respectively. $\mathbf{o}$ Summary box plots showing postnatal changes of the density of CX3CR1-positive cells in the IGL and ML in control (blue) and Csf1r-cKO (red) mice. The density of CX3CR1positive microglia was significantly lower in Csf1r-cKO mice than control mice at all postnatal days that we examined $(p<0.001$ at P7, $p<0.001$ at P11, $p=$ $4.21 \times 10^{-12}$ at $\mathrm{P} 14, p=2.01 \times 10^{-10}$ at P17, $p=1.26 \times 10^{-7}$ at $\left.\mathrm{P} 21\right) .{ }^{\star \star \star} p<0.001$. The density of microglia was measured in $11-19$ images containing the IGL and the ML in lobules IV-V of the cerebellar vermis $(256 \mu \mathrm{m} \times 256 \mu \mathrm{m})$ from three to five mice. Scale bars, $30 \mu \mathrm{m}(\mathbf{a}-\mathbf{I})$ and $10 \mu \mathrm{m}(\mathbf{m}, \mathbf{n})$

density slightly increased after P14, but was still $<30 \%$ of control mice (Fig. 4k, l, o). CX3CR1-positive cells observed after P14 in Csflr-cKO mice were ameboid, possessing larger cell bodies and thicker, shorter and less ramified processes than controls (Fig. $4 \mathrm{~m}, \mathrm{n}$ ), which was consistent with observations in Csf1r-null KO mice $\mathrm{e}^{35}$. Meanwhile, expression patterns of an astrocyte marker (glial fibrillary acidic protein, GFAP), a Bergmann glia marker (3phosphoglycerate dehydrogenase, 3PGDH) and a myelinating oligodendrocyte marker (myelin basic protein, MBP) in Csf1rcKO mice were identical to those in control mice at P22-P24 (Supplementary Fig. $6 \mathrm{j}-\mathrm{l}, \mathrm{n}-\mathrm{p}$ ). Taken together, these results suggest that microglia are selectively and severely deleted from the Csf1r-cKO cerebellum during early postnatal development.

CF elimination after P10-P12 is impaired in Csf1r-cKO mice. Using Csf1r-cKO mice, we examined the functional roles of microglia in the refinement of CFs. In young adult control mice (P21-P40), nearly $80 \%$ of PCs showed CF-EPSCs with a single step. In contrast, numbers of PCs with multiple CF-EPSC steps were significantly increased in Csflr-cKO mice, suggesting that developmental regression of CFs was impaired (Fig. 5a). CFEPSCs recorded in Csflr-cKO mice showed slightly larger amplitudes and faster rise times, but the paired-pulse ratio and decay time constant were normal (Supplementary Table 2). The disparity index and disparity ratio, values that represent strengthening of the most predominant $\mathrm{CF}$ relative to other weaker ones, were normal in Csflr-cKO mice (Supplementary Table 2). We checked the effect of Ibal deletion on CF elimination using Iba1(iCre/iCre) mice (Iba1-KO, Fig. 5a). The density of microglia in Iba1-iCre mice was not significantly different from that in control mice at P21 (Supplementary Fig. 7), and CF elimination was normal in these mice (Fig. 5a). The distribution histograms for Csf1r-cKO mice at P60-P80 (Fig. 5b) were virtually identical to those of young adult mice (P21-P41, Fig. 5a), suggesting that the impairment of CF elimination persisted into adulthood.

Next, we examined the developmental course of CF elimination in Csf1r-cKO mice. At P6-P8, most PCs were innervated by three or more CFs in both Csf1r-cKO and control mice (Fig. 5c), which suggests that formation of CF synapses was normal in Csflr-cKO mice. From P10-P12, the impairment of CF synapse elimination started to become significant (Fig. 5d). CF synapse elimination progressed weakly from $\mathrm{P} 13-\mathrm{P} 15$ to $\mathrm{P} 16-\mathrm{P} 18$, even in the Csf1rcKO mice, but stopped without reaching the control level (Fig. 5e, f). These results indicate that microglia-dependent CF elimination starts from P10 to P12. This postnatal period is almost coincident 
a

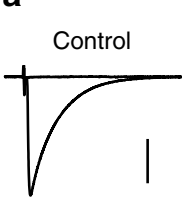

$\mathrm{P} 21-\mathrm{P} 40$
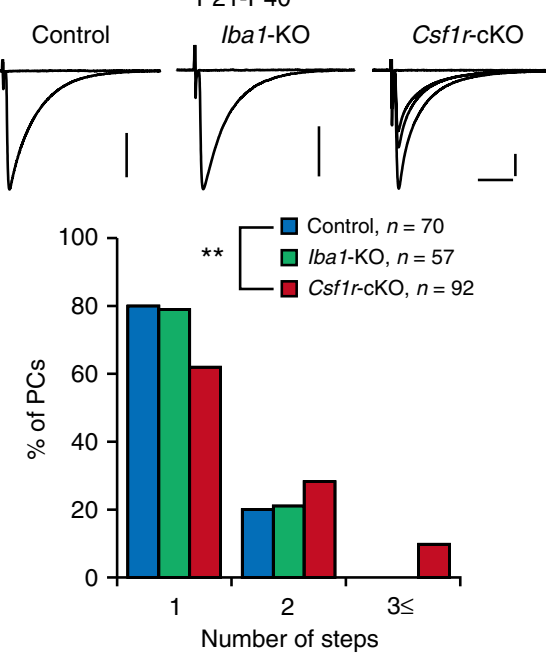

b

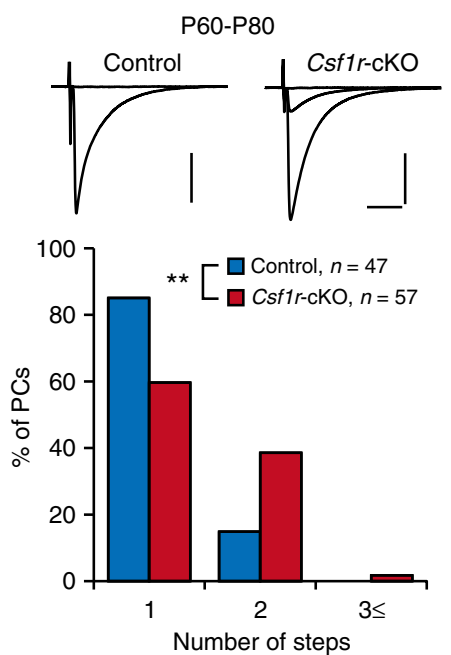

c
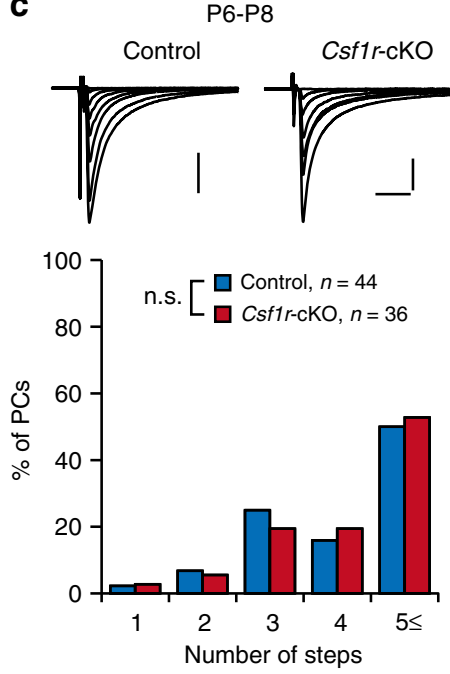

d
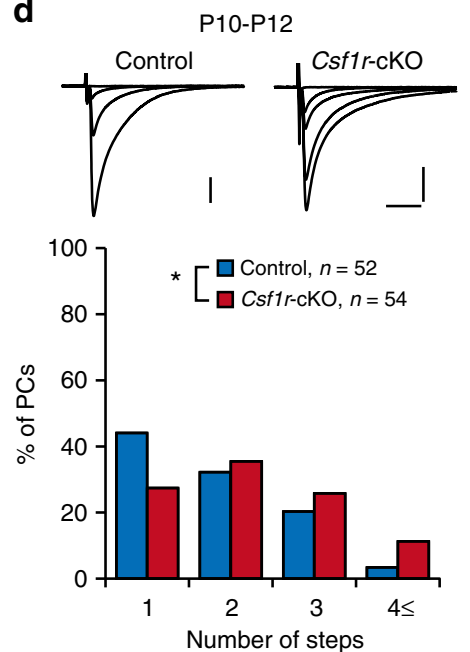
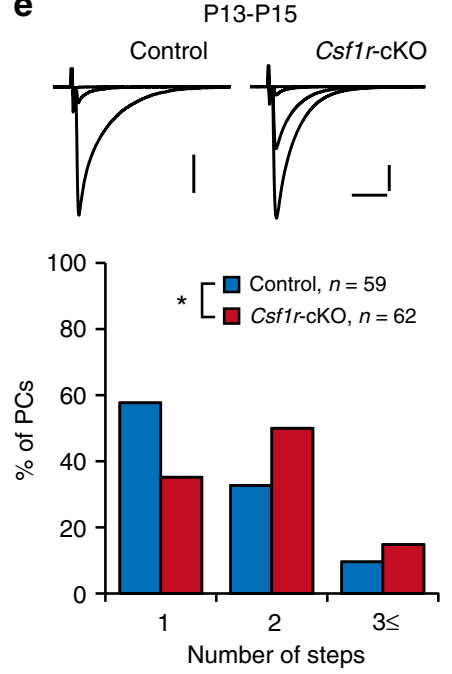

f
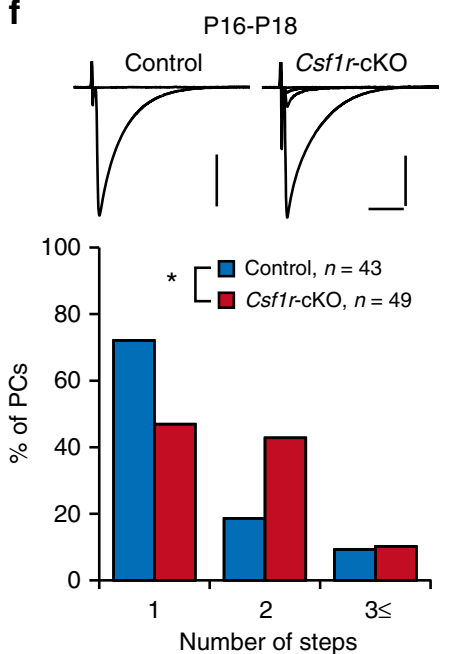

Fig. 5 CF synapse elimination after P10-P12 is impaired in Csf1r-CKO mice. a (upper) Representative traces of CF-EPSCs in control (left), Iba1-KO (middle), and Csf1r-cKO (right) PCs in response to electrical stimulation with gradually increasing stimulus intensities; $V h=-10 \mathrm{mV}$. (lower) Frequency distribution histograms showing the number of discrete CF-EPSC steps in control (blue), Iba1-KO (green), and Csf1r-cKO (red) mice aged P21-P40. PCs were evenly sampled from all lobules. Data are sampled from four control, four Iba1-KO, and five Csf1r-cKO mice. CF synapse elimination was normal in Iba1-KO mice ( $p=0.887$, Mann-Whitney $U$ test) but significantly impaired in Csf1r-cKO mice $(p=0.007)$. b Frequency distribution histograms of the number of CFs in control (blue) and Csf1r-cKO (red) mice at P60-P80 ( $p=0.004)$. c-f Developmental course of CF synapse elimination. Frequency distribution histograms of the number of CFs at P6-P8 (c, $p=0.207)$, P10-P12 (d, $p=0.034), \mathrm{P} 13-\mathrm{P} 15$ (e, $p=0.028)$, and P16-P18 (f, $p=0.030)$. Data were obtained from 4 to 5 control and 4 to 5 Csf1r-cKO mice at each postnatal period. Scale bars, $1 \mathrm{nA}$ and $10 \mathrm{~ms}$. Vh $=-70 \mathrm{mV}(\mathbf{c})$ and $-10 \mathrm{mV}(\mathbf{b}, \mathbf{d}-\mathbf{f}) .{ }^{\star} p<0.05 ;{ }^{\star \star} p<0.01 ; \mathrm{n} . \mathrm{s}$., $p>0.05$

with the critical period identified from the experiments using liposomal clodronate (Fig. 2) if the delay of its pharmacological effect is taken into account.

Microglia rarely engulf CFs during postnatal development. We next investigated how microglia promoted CF synapse elimination. Microglia are thought to refine developing neuronal circuits by engulfing surplus synaptic structures ${ }^{19-23}$. Therefore, we assessed the microglial engulfment of CFs by double staining of microglia and CFs. CFs were anterogradely labeled with Alexa Fluor 568-conjugated dextran, and microglia were immunostained with Ibal antibody in C57BL/6 mice at P10-P12. In the present study, labeled CF fragments that were completely internalized in the Iba1-labeled microglial cytoplasm were regarded as engulfed CFs. However, CF varicosities connected to the main CFs with fine processes were not regarded as engulfed, no matter how closely associated they were. Some Iba1-labeled microglial processes were closely adjacent to Alexa Fluor 568-labeled CFs around PC somata (arrows, arrowheads, and double arrowheads in Fig. 6a-f). However, these belonged to passing CFs or collateral boutons emerging from other CF branches, suggesting that they were not completely internalized in microglia (Fig. 6b). We quantified a proportion of the microglia with inclusions of isolated $\mathrm{CF}$ fragments in $\mathrm{C} 57 \mathrm{BL} / 6$ mice. For this analysis, only microglia that associated with labeled CFs in the PCL or ML were analyzed. We found only 2 out of 47 microglia (three mice) with inclusion of CF fragments (Fig. 6g). All inclusions were observed in the soma, not in the processes of microglia. These data do not completely rule out the possibility of engulfment of CFs but suggest that the incidence is very rare, even if microglia are closely associated with labeled CFs.

The Alexa Fluor 568-conjugated dextran is thought to be relatively resistant to lysosomal activity ${ }^{36}$, but it might be rapidly 

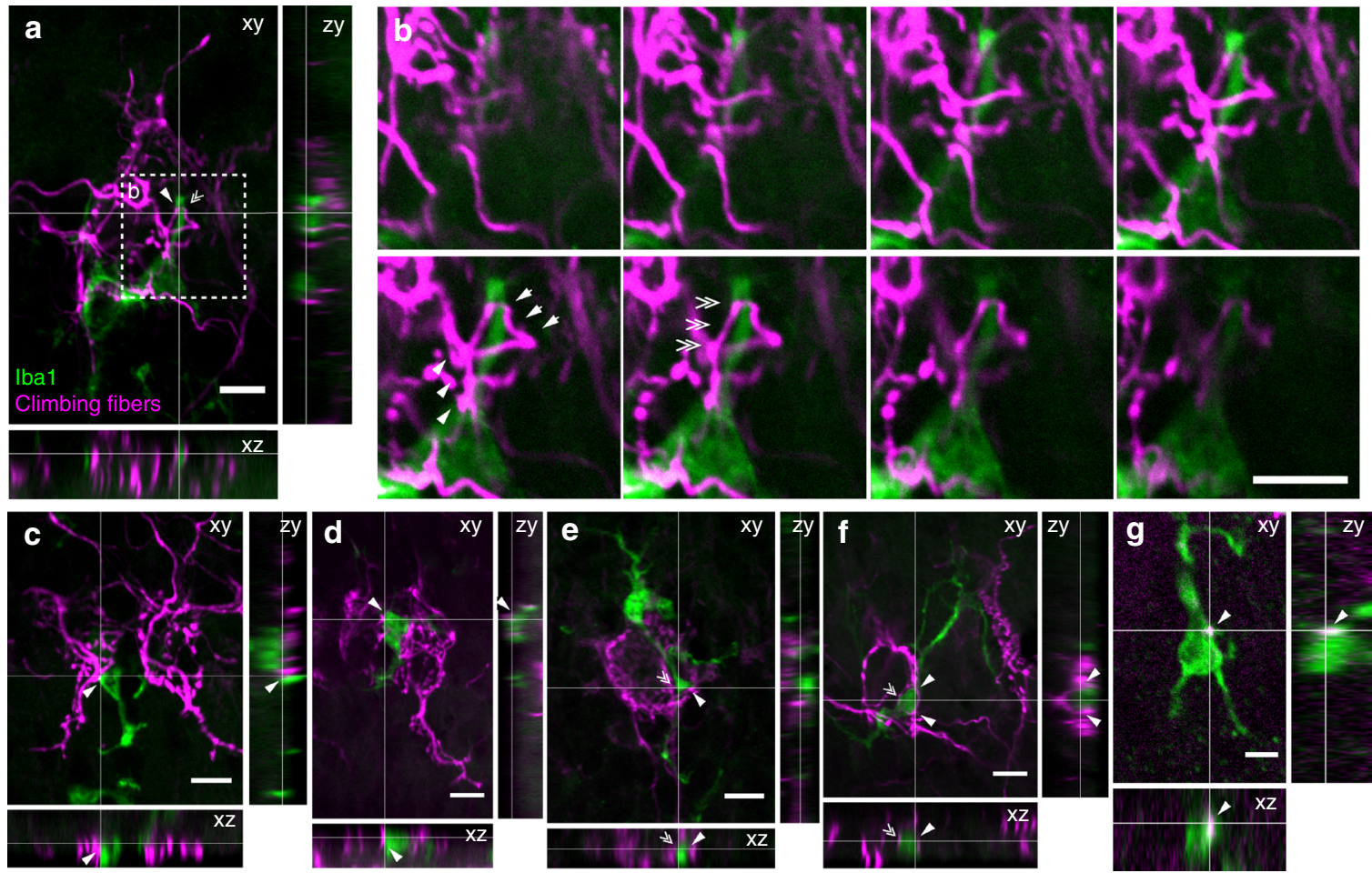

C57BL/6 mice + 3-AP, harmaline, nicotinamide
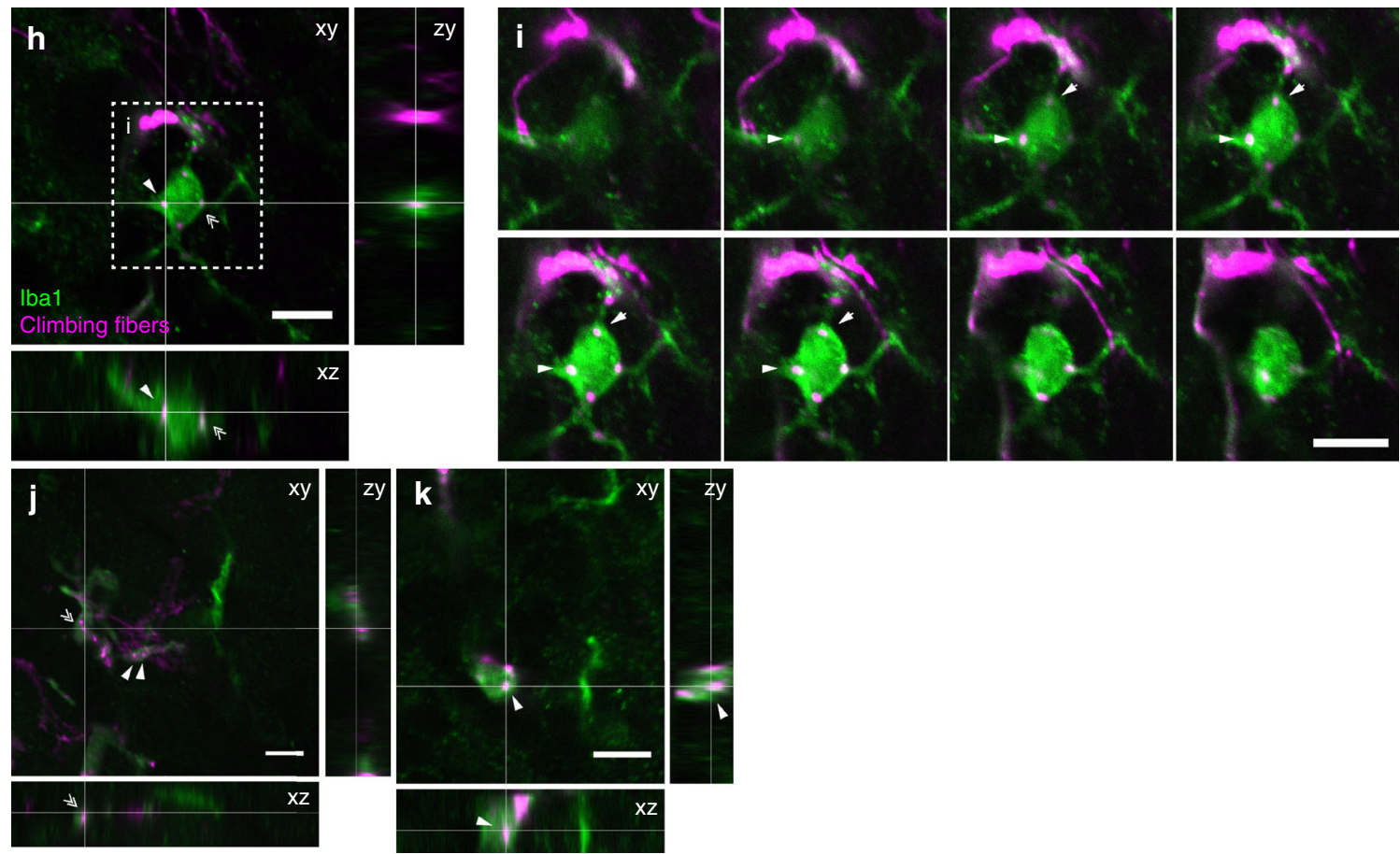

Fig. 6 Double staining of CFs and microglia in the developing cerebellum. a Orthogonal fluorescence images of Iba1-positive microglia (green) and CFs anterogradely labeled with Alexa Fluor 568-dextran (magenta) in C57BL/6 mice. The $x y$-image is a single plane with its position represented as white lines in the $x z$ and $z y$ images. $\mathbf{b}$ Serial enlarged images $(\Delta z=1 \mu \mathrm{m}$, from the left top to the right bottom) of a region surrounded by a dotted line in (a). Alexa Fluor 568-labeled CFs were very closely associated with Iba1-positive microglial processes but were not internalized in microglia (arrows, arrowheads, and double arrowheads). c-f Orthogonal fluorescence images of other microglia. $\mathbf{g}$ One of the rare examples in which an isolated CF fragment was internalized in Iba1-positive microglia. $\mathbf{h}$ Orthogonal fluorescence images similar to a but taken from C57BL/6 mice administrated 3-AP, harmaline and nicotinamide. i Serial enlarged images of a region surrounded by a dotted line in $\mathbf{h} . \mathbf{j}$, $\mathbf{k}$ Orthogonal fluorescence images of other 3-AP treated microglia. Fragments of Alexa Fluor 568-labeled CFs are included in Iba1-positive microglia (arrows and arrowheads in $\mathbf{h}-\mathbf{k}$ ), indicating that the fluorescence of Alexa Fluor 568 can be detected after being engulfed by microglia. Scale bars, $10 \mu \mathrm{m}$ 
degraded and become undetectable in our system. To test this possibility, Alexa Fluor 568-labeled CFs were partially degenerated by sequential administration of 3-acetylpyridine (3-AP), harmaline, and nicotinamide to mice ${ }^{37-39}$. As an analog of nicotinamide, 3-AP severely damages inferior olive (IO) neurons projecting CFs by metabolic disturbance. Co-administration of nicotinamide with 3 -AP protects the rest of the central nervous system from extensive lesions ${ }^{38,40,41}$. Harmaline produces over-activation of the IO neurons ${ }^{38,41}$, which apparently accelerates the metabolic changes in this nucleus. Coadministration of 3-AP with nicotinamide and harmaline locally restricts the central lesioned area to the $\mathrm{IO}^{38}$. In these drugtreated mice, Alexa Fluor 568-labeled CF fragments that were clearly isolated from labeled CFs and internalized in microglia (Fig. 6i) were frequently observed (25 of 33 microglia) (arrows and arrowheads in Fig. 6h-k), which indicates that Alexa Fluor 568 could be detected after being engulfed by microglia. Taken together, these results suggest that majority of surplus CFs are not removed by direct engulfment by microglia in the developing cerebellum.

PF-PC synapses are normal in Csflr-cKO mice. We further explored mechanisms of microglia-dependent CF synapse elimination. It has been well-established that proper formation of PF-PC synapses and activation of the mGluR1-mediated signaling cascade at synapses are crucial for CF synapse elimination $^{15,28}$. Therefore, microglia may indirectly affect CF elimination by promoting PF-PC synapse formation and/or function. To test this possibility, we examined the generation of functional PF-PC synapses at P16-P18 in Csflr-cKO mice. The paired-pulse ratio (Fig. 7a) of PF-EPSCs was not different between Csflr-cKO and control mice, which suggests that the presynaptic functions of PFs are normal in Csflr-cKO mice. To examine the density of functional PF synapses, we recorded miniature excitatory postsynaptic currents (mEPSCs). Frequency and amplitude of mEPSCs with 10-90\% rise times longer than 1 $\mathrm{ms}$, which mainly arise from PF synapses ${ }^{42}$, were analyzed. We found that the amplitude and frequency of mEPSCs in Csf1r-cKO mice were not significantly different from those in control mice (Fig. 7d-f). Moreover, the stimulus-response curve of PF-EPSCs was measured. Amplitudes of PF-EPSCs increased with stimulus intensity similarly in control and Csf1r-cKO PCs (Fig. 7b). These results suggest that the density of functional PF-PC synapses is normal in Csflr-cKO mice. This was also morphologically confirmed by co-immunostaining for vesicular glutamate transporter 1 (VGluT1), a marker of PF terminals, and calbindin, a marker for PCs (Fig. 7g-l). VGluT1-positive puncta were closely associated with calbindin-labeled PC spines in both control and Csfr1cKO mice (Fig. 7g-1), and the overall signal intensity of VGluT1 in the ML was unchanged in the Csf1r-cKO mice (Supplementary Fig. $8 g, j, i)$.

Because activation of mGluR1 signaling at PF synapses is crucial for the late-phase CF synapse elimination ${ }^{15}$, we next assessed abnormalities in mGluR1 signaling at PF synapses. Stimulus-response curves (Fig. 7c) and half-width of mGluR1mediated PF-EPSCs evoked by tetanic stimulus of PFs were identical between control and Csf1r-cKO mice (Control: $290.8 \pm$ $26.0 \mathrm{~ms}, n=17$ cells from six mice; Csf1r-cKO: $268.2 \pm 27.6 \mathrm{~ms}$, $n=14$ cells from four mice, $p=0.557$ by $t$ test). Immunofluorescence of mGluR1a was co-localized with that of calbindin at spines in both control and Csf1r-cKO mice (Supplementary Fig. 8a-f). Signal intensities of mGluR1 $\alpha$ immunofluorescence in the ML were also identical between control and Csf1r-cKO mice (Supplementary Fig. $8 \mathrm{~h}, \mathrm{k}, \mathrm{l}$ ). Taken together, these results indicate that formation of PF-PC synapses and activation of
mGluR1-mediated signaling are not altered in Csf1r-cKO mice. The impairment of CF elimination in the Csf1r-cKO mice was therefore not a result of abnormalities in the formation or function of GC-PF-PC synapses.

Inhibitory transmission is reduced in Csflr-cKO mice. In addition to the PF-PC synapses, we have previously demonstrated that formation and activation of GABAergic inhibitory synapses on PCs during the second postnatal week are also crucial for CF synapse elimination ${ }^{29}$. Therefore, it was possible that microglia affect CF elimination by promoting inhibitory synapse formation on PCs. To test this possibility, we examined miniature inhibitory postsynaptic currents (mIPSCs) and found that generation of mIPSCs was severely impaired at P10-P12 in Csf1rcKO mice (Fig. 8). Cumulative frequency distributions of mIPSC amplitudes significantly shifted to lower amplitudes in the Csf1rcKO mice compared with control mice (Fig. 8a-c). The average amplitude of mIPSCs was significantly smaller and their frequency was significantly lower in Csf1r-cKO mice than in control mice (Fig. 8d, e). Consistent with alterations in mIPSCs, signal intensity of the vesicular GABA transporter (VGAT), a marker of inhibitory synaptic terminals, was significantly decreased in the PCL, the GC, and the ML in Csflr-cKO mice (Fig. 8f-j). However, the density of VGAT-positive puncta in the PCL was not changed in the Csf1r-cKO mice (Control: $0.024 \pm 0.005 \mu \mathrm{m}^{-3}, n=3$ mice; Csf1r-cKO: $0.020 \pm 0.001 \mu \mathrm{m}^{-3}, n=4$ mice, $p=0.234$ by $t$ test), suggesting that morphogenesis of inhibitory synapses is largely normal but inhibitory synaptic transmission is impaired in Csf1rcKO mice. These results indicate that microglia are crucial for the formation of functional inhibitory synapses on PCs during postnatal cerebellar development.

Diazepam restores CF synapse elimination in Csflr-cKO mice. The results described above raise the possibility that impairment of CF elimination is caused by reduction of inhibitory synaptic transmission on $\mathrm{PCs}^{29}$. To test this possibility, we examined whether the administration of diazepam, a sensitizer of $\mathrm{GABA}_{\mathrm{A}}$ receptors, restored CF synapse elimination (Fig. 9). We previously demonstrated that impairment of CF synapse elimination in Gad67-defective heterozygous mice was restored by administration of diazepam, which suggests that developmental abnormalities caused by decreased GABA release can be recovered by the augmentation of postsynaptic $\mathrm{GABA}_{\mathrm{A}}$ receptors ${ }^{29,43}$. We first confirmed that acute application of diazepam $(1 \mu \mathrm{M})$ to slices prepared from Csflr-cKO mice increased the amplitude of mIPSCs (Fig. 9a-d). Diazepam or vehicle solution was injected intraperitoneally into $C s f 1 r$-cKO mice once per day from P9 to P12. The frequency distribution histograms of CF numbers in vehicle-treated Csflr-cKO mice was not different from that of naïve Csf1r-cKO mice (Fig. 5a; Fig. 9e-g). Importantly, the number of PCs innervated by a single CF was significantly increased in diazepam-treated Csf1r-cKO mice (Fig. 9g). This result indicates that impairment of CF synapse elimination in Csf1r-cKO mice is mainly caused by impaired inhibitory synaptic transmission to PCs during P9-P12.

\section{Discussion}

As shown herein and reported previously ${ }^{2-4,7}$, microglia are mainly distributed in the WM in the cerebellum at birth and they extensively translocate to the cerebellar cortex after P7, mainly from the WM (Fig. 1) ${ }^{4}$. In this study, we found that microglia transiently show biased distribution from the PCL to the IGL that peaked beneath the PCL at P8-P9. This postnatal period is coincident with the start of CF synapse elimination in the mouse cerebellum $^{33}$, suggesting the contribution of microglia to this 
a
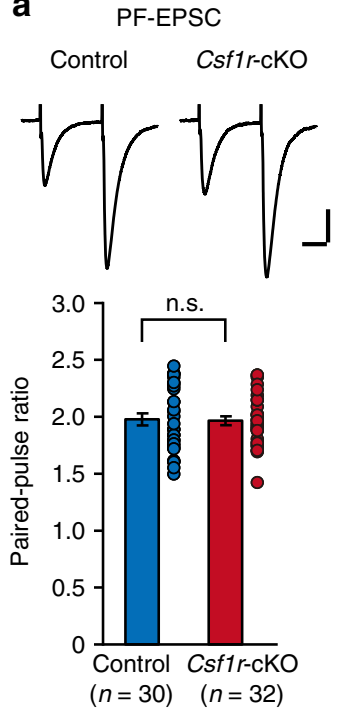

b PF-EPSC (AMPAR) Control Csf1r-cKO
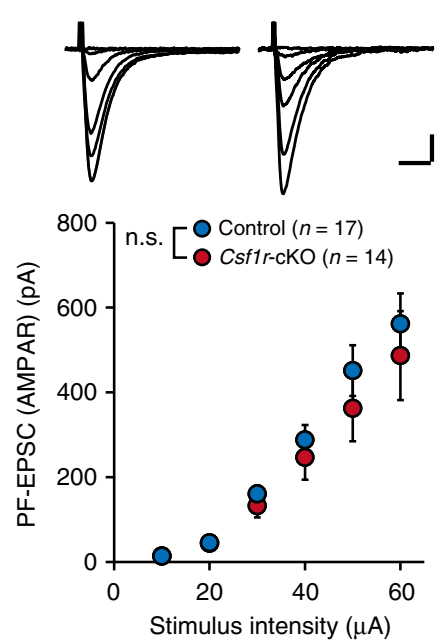

C PF-EPSC (mGluR)
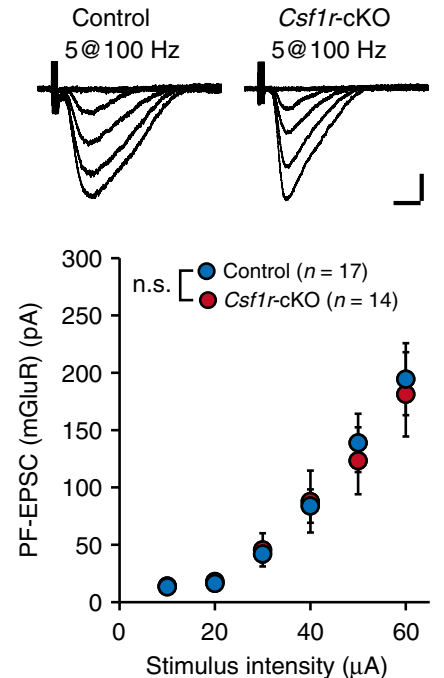

d
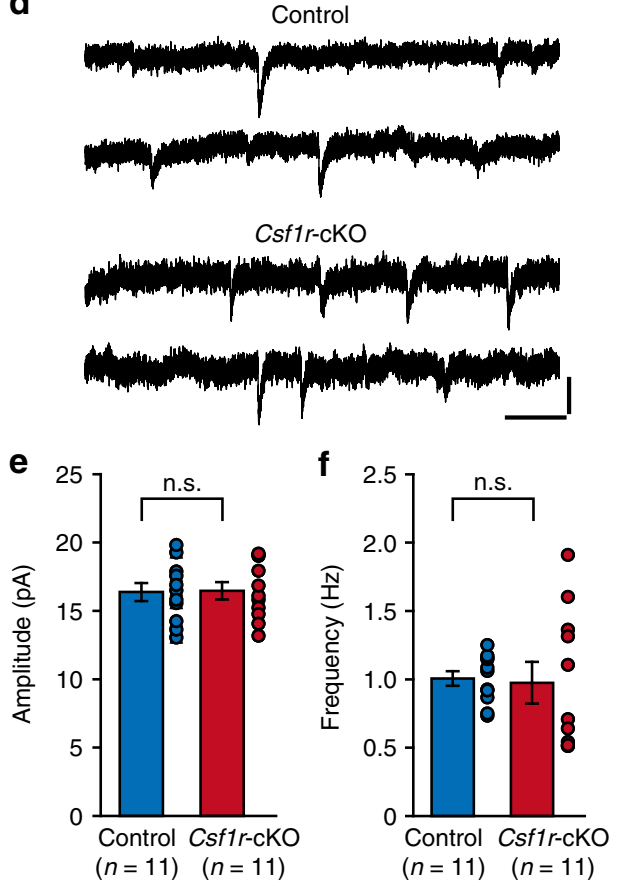
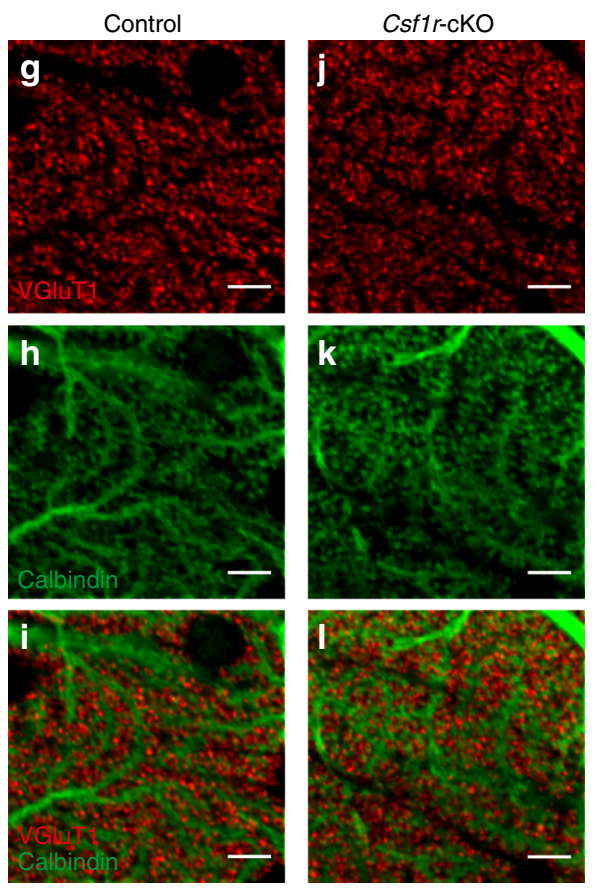

Fig. 7 Parallel fibers to PC synapses are normal in Csf1r-cKO mice. a (upper) PF-EPSCs recorded in a control or a Csf1r-cKO PC in response to paired stimuli with an interval of $50 \mathrm{~ms}$. (lower) The paired-pulse ratio was not different between control ( $n=30$ cells from seven mice) and Csf1r-cKO ( $n=32$ cells from eight mice, $p=0.972$, Mann-Whitney $U$ test). b (upper) PF-EPSCs evoked by stimulus intensities of 10, 20,30, 40, 50, and $60 \mu \mathrm{A}$ in a control or a Csf1rcKO PC. (lower) Stimulus-response curve of PF-EPSCs. The peak amplitudes of PF-EPSCs were measured and plotted against stimulus intensity. No difference is observed between control ( $n=17$ cells from six mice) and Csf1r-cKO ( $n=14$ cells from four mice, $p=0.459$, two-way RM ANOVA). c (upper) Traces of mGluR1-mediated currents evoked by tetanic stimulation (five pulses at $100 \mathrm{~Hz}$ ) with 10, 20,30,40,50, and $60 \mu \mathrm{A}$ stimulus intensities. (lower) The stimulus-response curves of mGluR1-mediated currents. These data were obtained from the same cells shown in (b). No difference was observed ( $p$ $=0.886$, two-way RM ANOVA). d Miniature EPSCs (mEPSCs) recorded from a control or a Csf1r-cKO PC in the presence of $1 \mu \mathrm{M}$ TTX and $100 \mu \mathrm{M}$ picrotoxin. Amplitude (e, $p=0.921, t$ test) or frequency $(\mathbf{f}, p=0.555$, Mann-Whitney $U$ test) did not differ between control ( $n=11$ cells from three mice) and Csf1r-cKO ( $n=11$ cells from three mice) mice. Vh $=-70 \mathrm{mV}$ (a-f). Electrophysiological data were obtained from PCs in lobules IV-V at P16-P18 (a-f). Scale bars, 100 pA and $20 \mathrm{~ms}(\mathbf{a}, \mathbf{c}), 200$ pA and $10 \mathrm{~ms}$ (b), and 10 pA and $200 \mathrm{~ms}$ (d). n.s., p > 0.05. g-I Immunostaining for VGluT1 (g, j), calbindin (h, k), and merged of them $(\mathbf{i}, \mathbf{I})$. Images were taken from the ML in lobules IV-V in the vermis. Scale bars, $5 \mu \mathrm{m}$. PC dendrites possessed dense spines that faced VGluT1 puncta in both control and Csf1r-cKO mice. Data are presented as mean \pm SEM

event. In fact, this period was temporally coincident with the time window when clodronate liposome injection was effective (Fig. 2) and when the impairment of synapse elimination in Csf1r-cKO began (Fig. 5). These lines of evidence suggest that initiation of microglia-dependent cerebellar circuit maturation has a close relationship with the transient accumulation of microglia around the PCL-IGL area.

Microglia are absent from the CNS in Csf1r-null $\mathrm{KO}$ mice ${ }^{1,34,35}$, and pharmacological inhibition of CSF1R clears $\sim 99 \%$ of microglia from the brain ${ }^{44}$. Therefore, it is widely believed that 
a

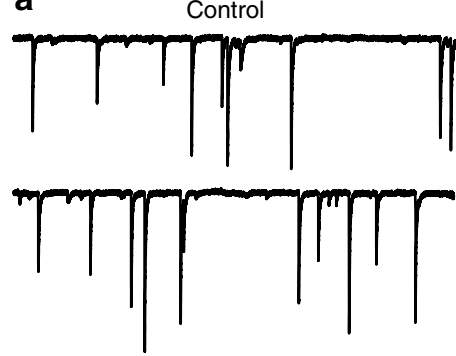

C

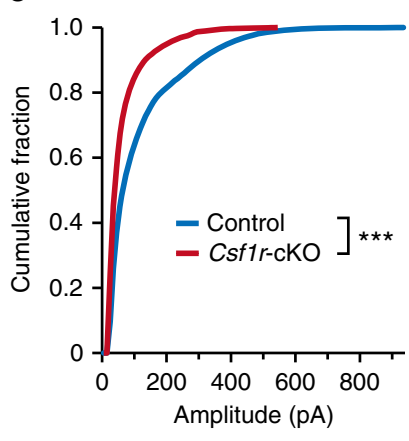

b

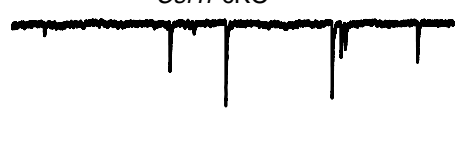

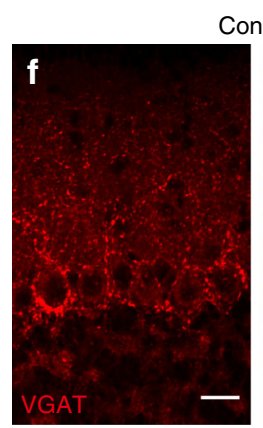

Control

d

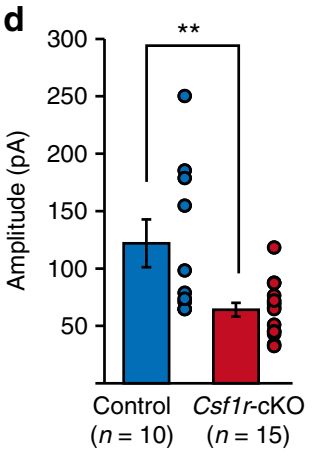

e

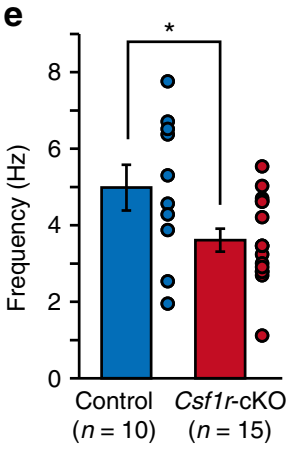

Csf1r-cKO
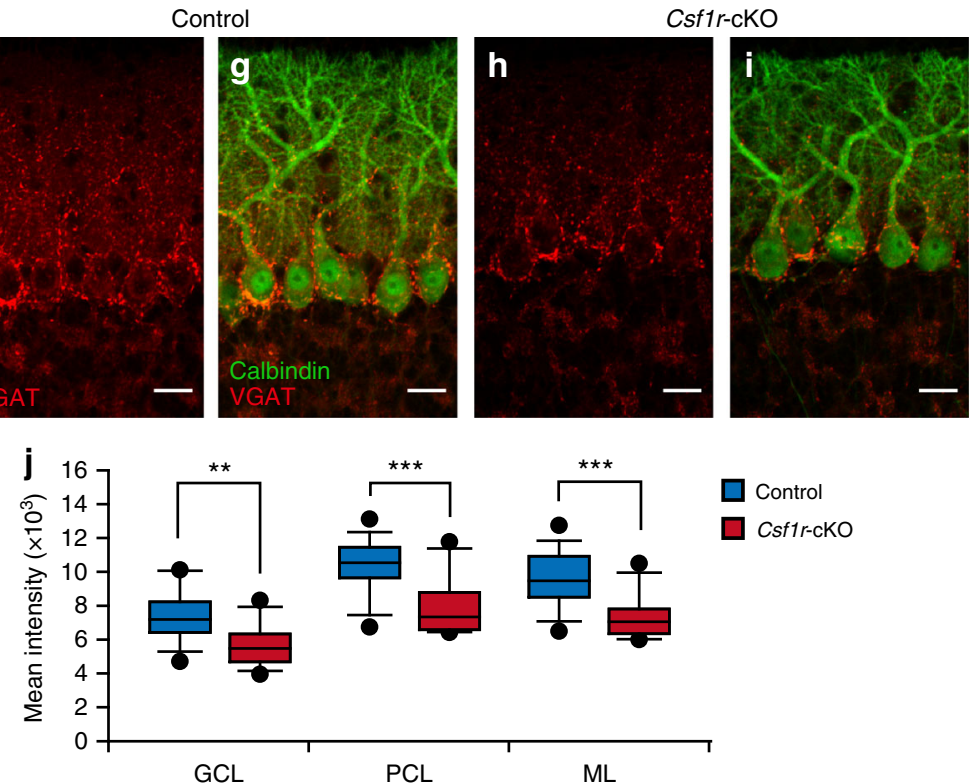

Control

$\square$ Csf1r-cKO

Fig. 8 Generation of functional inhibitory synapses is impaired in Csf1r-cKO mice. a, b Representative traces of mIPSCs recorded in a control (a) or a Csf1rcKO (b) PC in the presence of $1 \mu \mathrm{M}$ TTX and $10 \mu \mathrm{M}$ NBQX; Vh $=-70 \mathrm{mV}$. Scale bars, $100 \mathrm{pA}$ and $500 \mathrm{~ms}$. c Cumulative fractions of amplitudes of mIPSCs in control (blue) and Csf1r-cKO (red) mice. Recordings were obtained from PCs in lobules IV-V at P10-P12. The incidence of mIPSCs with smaller amplitudes is significantly increased in Csf1r-cKO mice ( $p<0.001$, Kolmogorov-Smirnov test). Graphs consist of 8426 events from 10 control cells from three mice and 9847 events from 15 Csf1r-cKO cells from six mice. d, e Averaged amplitude (d) and frequency (e) of mIPSCs. Amplitude was significantly smaller ( $\mathbf{d}, p=0.006$, Mann-Whitney $U$ test) and frequency was significantly lower (e, $p=0.013$ ) in Csf1r-cKO (red) than in control (blue) mice. Data were obtained from 10 cells from three control mice and 15 cells from six Csf1r-cKO mice. Data are presented as mean \pm SEM. f-i Immunostaining for VGAT $(\mathbf{f}, \mathbf{h})$, and merged images for VGAT (red) and calbindin (green) $(\mathbf{g}, \mathbf{i})$ in a control $(\mathbf{f}, \mathbf{g})$ and a Csf1r-cKO mouse (h, i) at P11. Images were taken from lobules IV-V in the vermis. Scale bars, $20 \mu \mathrm{m}$. $\mathbf{j}$ Mean fluorescence intensity of VGAT staining in the granule cell layer (GCL), the PC layer (PCL), and the molecular layer (ML) in lobules IV-V of cerebellar vermis. Signal intensity of VGAT was significantly lower in Csf1r-cKO mice than in control mice in all layers (GCL: $p=0.001 ; \mathrm{PCL}: p<0.001 ; \mathrm{ML}: p<0.001, t$ test). Each data set was obtained from 15 images $(256 \mu \mathrm{m} \times 256 \mu \mathrm{m}) \mathrm{containing} \mathrm{GCL,} \mathrm{PCL,} \mathrm{and} \mathrm{ML}$ from three control and three Csf1r-cKO mice at P10-P12. Scale bars, $30 \mu \mathrm{m}$. ${ }^{\star} p<0.05 ;{ }^{\star \star} p<0.01 ;{ }^{\star \star \star} p<0.001$

CSF1R is crucial for the proliferation, differentiation, and survival of microglia. CSF1R is mainly expressed in microglia in the brain, but some immunohistochemical analyses reported that it is also expressed in nestin-positive neural progenitor cells and subsets of neurons, including $\mathrm{PCs}^{45-47}$. Therefore, we generated microgliaselective conditional KO mice by crossing Csflr-floxed and Iba1-
iCre mice to abolish microglia from the brain. Csf1r-null $\mathrm{KO}$ mice rarely survive beyond $\mathrm{P} 21$ and exhibit severe abnormalities in brain architecture, such as a small brain size, enlarged ventricles, a thinned cortex, defects in midline crossing of callosal axons, and a loss of microglia ${ }^{34,35}$. At least some of these abnormalities in brain structures are likely caused by the deletion of Csf1r from 
a

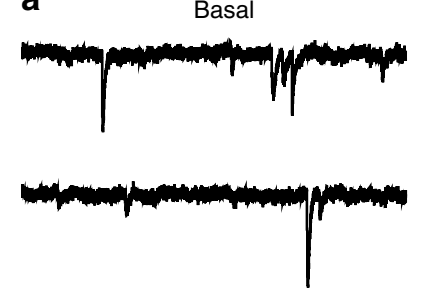

C

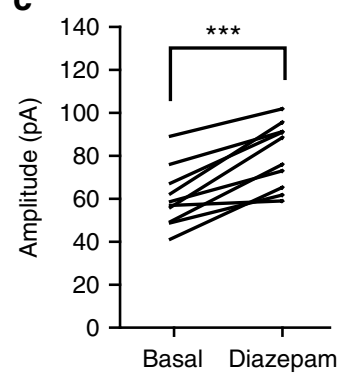

b

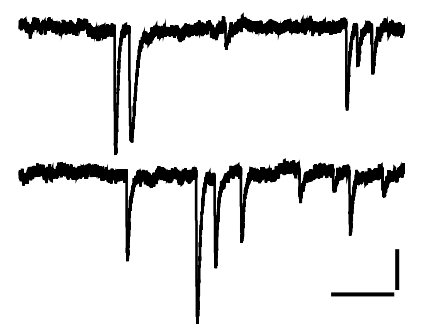

d

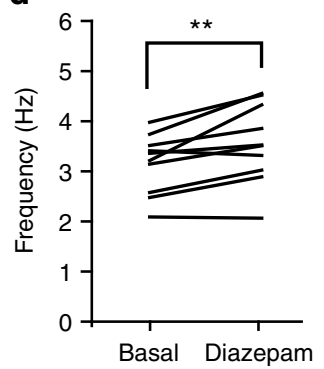

e
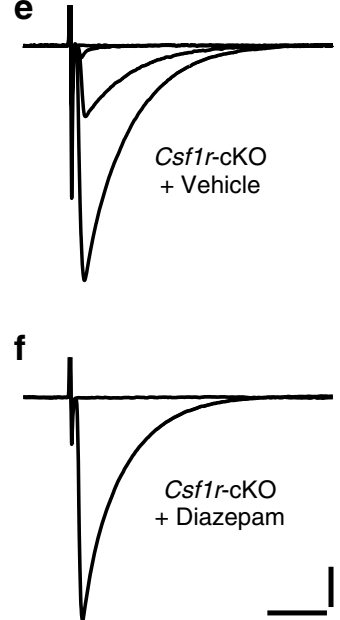

g

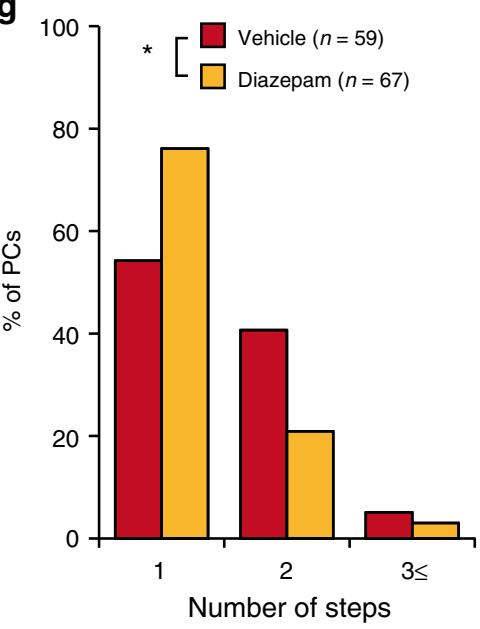

Fig. 9 Administration of diazepam restores CF elimination in Csf1r-cKO mice. a-d Representative traces of mIPSCs recorded from a Csf1r-cKO PC before (a) and after (b) application of $1 \mu \mathrm{M}$ diazepam. $\mathrm{Vh}=-70 \mathrm{mV}$. Scale bars, $30 \mathrm{pA}$ and $250 \mathrm{~ms}$. Mean amplitude of mIPSCs significantly increased during bath application of diazepam (c, $p<0.001$, paired $t$ test, $n=10$ cells from three mice). Frequency was also significantly increased by the diazepam application ( $\mathbf{d}, p=0.006)$. e, $\mathbf{f}$ Representative traces of CFEPSCs in Csf1r-cKO mice treated with vehicle (e) or diazepam (f); $\mathrm{Vh}=$ $-10 \mathrm{mV}$. Scale bars, $500 \mathrm{pA}$ and $10 \mathrm{~ms}$. g Frequency distribution histograms showing the number of CFs innervating each PC in vehicle- and diazepam-treated Csf1r-cKO mice. Data were sampled from five vehicletreated and five diazepam-treated mice. The distribution of the histogram of vehicle-treated Csf1r-cKO mice is virtually identical to that of naïve Csf1rcKO mice ( $p=0.540$, Mann-Whitney $U$ test). The percentage of monoinnervated PCs is significantly increased in diazepam-treated Csf1r-cKO mice $\left(p=0.011\right.$, Mann-Whitney $U$ test). ${ }^{\star} p<0.05 ;{ }^{\star \star} p<0.01 ;{ }^{\star \star \star} p<0.001$

non-microglial cells, because similar abnormalities are partially reproduced in mice in which Csf1r is conditionally deleted from nestin-positive neural progenitor cells ${ }^{45}$. Our analysis also supports these data. Csflr-cKO mice showed severe reduction in microglial numbers in the developing cerebellum (Fig. 4), which confirms that Csf1r in microglia is crucial for survival of microglia in the brain. However, Csf1r-cKO mice survived to adulthood and were fertile. The abnormalities in brain architecture reported in Csfir-null mice were not observed, except for the enlarged

ventricular size (Supplementary Fig. 5). Taken together, CSF1R signaling in microglia and non-microglial cells likely has distinct roles in the formation and maintenance of brain architecture. In the Csflr-cKO mice, Csflr would also be deleted from peripheral macrophages ${ }^{48}$. Because peripheral immune responses are thought to modulate gene expression and synaptic functions in the brain ${ }^{49}$, deletion of Csflr in peripheral macrophages might affect CF synapse elimination. However, impairment of CF synapse elimination was reproduced by deletion of microglia using intracerebellar injection of liposomal clodronate (Fig. 2), indicating that microglia within the cerebellum play the dominant role in circuit refinement.

Our electrophysiological and morphological analyses suggested that GABAergic transmission on PCs was significantly impaired in Csf1r-cKO mice (Fig. 8). Molecular mechanisms for microgliadependent facilitation of development of inhibitory synapses in the cerebellum are currently unknown but brain-derived neurotrophic factor (BDNF)-tyrosine receptor kinase B (TrkB) signaling is a candidate. BDNF is produced by and released from microglia ${ }^{18}$, the amplitude and frequency of mIPSCs in PCs are reduced in $\operatorname{TrkB}-\mathrm{KO}$ mice $^{50}$, and the number of GAD65-positive boutons is reduced in cerebellum-specific TrkB-KO mice ${ }^{51}$. Importantly, CF synapse elimination is also impaired in mutant mice with defective BDNF-TrkB signaling $g^{50,52,53}$. Cytokines might also be candidates. Interleukin- $1 \beta$ (IL-1 $\beta$ ) enhances plasma membrane insertion of $\mathrm{GABA}_{\mathrm{A}}$ receptors in cultured neurons and oocytes ${ }^{54}$, and in vivo nanoinjection of tumor necrosis factor $\alpha(\mathrm{TNF} \alpha)$ induces $\mathrm{GABA}_{\mathrm{A}}$ receptor trafficking to synaptic membranes in spinal cord neurons ${ }^{55}$. Identification of microglia-derived factors regulating inhibitory synaptic transmission on PCs will need to be identified in future studies.

CF synapse elimination during postnatal development is finely regulated by neuron-neuron interactions ${ }^{15,33}$. Many previous reports have demonstrated that $\mathrm{CF}$ synapse elimination is regulated by PF-PC synaptic activities. CF synapse elimination is impaired in animals that have abnormalities in the generation of PF-PC synapses ${ }^{15,26,33}$. PF synapses are thought to have roles in restricting the $\mathrm{CF}$ innervation sites to the proximal dendrites and in activating the mGluR1 signaling cascade crucial for late-phase CF elimination. In addition, CF elimination is also regulated by interneuron-PC interactions ${ }^{29}$. CF elimination is impaired in Gad67 heterozygous KO mice and this impairment is reversed by diazepam ${ }^{29}$. Activation of GABAergic synapses is thought to regulate $\mathrm{CF}$ synapse elimination, presumably by affecting intracellular calcium transients associating with $\mathrm{CF}$ inputs ${ }^{29,56}$. In the present study, we found that formation of functional PF synapses and activation of the mGluR1 signaling cascade were normal, but inhibitory synaptic transmission was severely impaired in the Csfir-cKO mice. The impairment of CF synapse elimination in Csflr-cKO mice was recovered by administration of diazepam, which suggests that the defect in GABAergic inhibition to PCs causes impairment of $\mathrm{CF}$ synapse elimination. In Csflr-cKO mice, regression of CF synapses was impaired from P10 to P12. It weakly proceeded until P14 but stopped thereafter (Fig. 5). This developmental course of CF regression in Csflr-cKO mice was very similar to that observed in Gad67 heterozygous KO mice ${ }^{29}$. These lines of evidence suggest that microglia indirectly affect $\mathrm{CF}$ synapse elimination by regulating GABAergic inhibition in the developing cerebellum. This notion is also supported by the rare observation of CFs internalized in microglia in the developing cerebellum (Fig. 6), which suggests that CF synapses are not generally removed by direct engulfment by microglia. However, because activated microglia have been reported to induce axonal retraction by expressing contact-dependent repulsive axon guidance molecules ${ }^{57,58}$, it remains a possibility that microglia also 
induce $\mathrm{CF}$ retraction by mechanisms not dependent on engulfment.

In conclusion, our present study revealed that microglia primarily facilitate formation of functional inhibitory synapses in the developing cerebellum, thereby promoting elimination of surplus CFs by driving mechanisms inherent in PCs. Microglia-neuron and neuron-neuron interactions cooperatively participate in postnatal circuit refinement in the cerebellum.

\section{Methods}

Animals. All experiments were conducted according to the guidelines of the experimental animal ethics committees (A16-137, M-P12-138, SA00041) and the biosafety committee for living modified organisms (28-223, 70, SD00800) of Hiroshima University, the University of Tokyo, and Niigata University, respectively. Male and female C57BL/6, Csf1r(lox/lox); Iba1(iCre/iCre), Csf1r(lox/lox); Ibal (+/+), Iba1(iCre/+); CAG-floxed STOP tdTomato and Iba1(iCre/iCre) mice aged from P5 to P80 were used for experiments. We generated Ibal-iCre (Fig. 3a-c) and CAG-floxed STOP tdTomato (Supplementary Fig. 4) mice. To generate Csflr(lox/ lox); Ibal(iCre/iCre) mice, we mated Csflr-floxed mice (Jackson Laboratories, stock no. 021212$)^{59}$ with Ibal-iCre mice. Genotypes of mice were examined by PCR of genomic DNA extracted from their tails. The following specific primers were used for Iba1-iCre mice: F, 5'-GGTGTCAGCAGAAGCTGATG-3'; R1, 5' AGCATCTTCCAGGTGTGTTC-3'; R2, 5'-CCTTCCCTACCCTGCAAATC-3' We maintained all mice in specific pathogen-free conditions on a reversed $12 \mathrm{~h}$ light/dark cycle (lights off at 8 PM) with free access to food and water.

Immunohistochemistry. Mice were deeply anesthetized by intraperitoneal injection of pentobarbital (100 mg kg-1 body weight) and transcardially fixed with 2 or $4 \%$ paraformaldehyde. Brains were removed, postfixed in the same fixative at $4{ }^{\circ} \mathrm{C}$ overnight, and stored in phosphate-buffered saline (PBS) containing $0.1 \% \mathrm{NaN}_{3}$. Parasagittal cerebellar sections of $50 \mu \mathrm{m}$ thickness were prepared from the vermis with a vibratome slicer (TDK-1000, Dosaka EM). The slices were incubated in $10 \%$ normal donkey serum, then in primary antibodies overnight at $4{ }^{\circ} \mathrm{C}$. Primary antibodies used in the present study were rabbit anti-Ibal (1:500, \#019-19741, Wako), mouse anti-CX3CR1 (1:100, \#149002, Biolegend), rabbit or mouse antiNeuN (1:500, \#ab177487, Abcam; 1:1000, \#MAB377, Merck), mouse anti-GFAP (1:500, \#MAB3402, clone GA5, Merck; 1:250, \#3670, Cell Signaling Technology), rabbit anti-NG2 (1:200, \#AB5320, Merck), chicken anti-myelin basic protein (1:100, \#AB9348, Merck), mouse anti-APC (1:250, \#NB600-1021, Novus Biologicals), rabbit anti-neurogranin (1:200, \#AB5620, Merck), goat or rabbit anticalbindin (1:200, \#Calbindin-Go-Af1040 or \#Calbindin-Rb-Se-1, Frontier Institute), rabbit anti-VGAT (1:200, \#VGAT-Rb-Af500, Frontier Institute), goat or guinea pig anti-parvalbumin (1:200, \#PV-Go-Af460; 1:500, \#PV-GP-Af1000, Frontier Institute), rabbit anti-mGluR1a (1:200, \#mGluR1a-Rb-Af811, Frontier Institute), and goat anti-VGluT1 (1:200, \#VGluT1-Go-Af310, Frontier Institute). After incubating in the primary antibodies, sections were washed and then incubated in Alexa Fluor 488-, Alexa Fluor 568-, Alexa Fluor 555- (1:500, Thermo Fisher Scientific, MA) or Alexa Fluor 488-, DyLight 405- or Cy3- (1:200, Jackson Immunoresearch) conjugated, species-specific secondary antibodies for $2 \mathrm{~h}$ at room temperature. Counter staining was performed with DAPI $\left(1 \mu \mathrm{g} \mathrm{ml} l^{-1}\right.$, Thermo Fisher Scientific) or Neuro Trace 530/615 Red or 500/525 Green Fluorescent Niss Stain (1:500, Thermo Fisher Scientific). We took images with a confocal laser scanning microscope (LSM700, Zeiss) or a fluorescent microscope (IX71, OLYMPUS) of immunostained slices from lobules IV-V because of their long straight alignment of cerebellar layers. We analyzed the images with ImageJ software (Rasband, W.S., ImageJ, U. S. National Institutes of Health, Bethesda, Maryland, USA, https://imagej.nih.gov/ij/, 1997-2016). When taking images of large regions, a KEYENCE BZ-X700 fluorescence microscope (KEYENCE) was used.

Production of transparent brain slices (SeeDB2). Mice were deeply anesthetized with pentobarbital (100 mg kg ${ }^{-1}$ body weight) and brains were fixed as described above. Parasagittal cerebellar sections of $500 \mu \mathrm{m}$ thickness were prepared from the vermis. The sections were cleared according to the SeeDB2 protocol ${ }^{32}$. Briefly, the sections were incubated with $2 \%$ saponin in PBS overnight. After immunohistochemistry against Ibal and calbindin, the sections were incubated serially with 33,50 , and $90 \%$ Omnipaque 350 (Daiichi Sankyo) in water containing 2\% saponin for $6-12 \mathrm{~h}$ each. Then the sections of lobules IV-V were immersed in $100 \%$ Omnipaque 350 and imaged with a confocal laser scanning microscope (FV1000, Olympus) equipped with $25 \times$ objective lens (XLPLN25XIMA, Olympus).

Electrophysiology. Mice aged P6-P80 were deeply anesthetized by $\mathrm{CO}_{2}$ inhalation and decapitated. The brains were quickly removed, and parasagittal slices with thickness of $250 \mu \mathrm{m}$ were prepared from cerebellar vermis with a vibratome slicer (VT1200S, Leica) in chilled normal artificial cerebrospinal fluid (ACSF) containing $125 \mathrm{mM} \mathrm{NaCl}, 2.5 \mathrm{mM} \mathrm{KCl}^{2} \mathrm{mM} \mathrm{CaCl}_{2}, 1 \mathrm{mM} \mathrm{MgSO}_{4}, 1.25 \mathrm{mM} \mathrm{NaH}_{2} \mathrm{PO}_{4}$,
$26 \mathrm{mM} \mathrm{NaHCO} 3$, and $20 \mathrm{mM}$ glucose, bubbled with $95 \% \mathrm{O}_{2}$ and $5 \% \mathrm{CO}_{2}$. After slice preparation, slices were kept at $25^{\circ} \mathrm{C}$ in normal ACSF.

Whole-cell recordings were made from visually identified PCs using an upright microscope (BX50WI, Olympus). PCs were equally sampled from all lobules except the experiment shown in Fig. 2, in which PCs within the liposome-injected lobule were selectively examined. All data were recorded at $32^{\circ} \mathrm{C}$ with an EPC10 patch clamp amplifier (Harvard Bioscience) without correction of liquid junction potential. Online data acquisition and offline data analysis were performed using Fit Master software (Harvard Bioscience) and the Mini Analysis Program (Version 6.0.7, Synaptosoft). The pipette solution for voltage clamp recordings was composed of $60 \mathrm{mM} \mathrm{CsCl}, 10 \mathrm{mM} \mathrm{Cs}_{\mathrm{D}}$-gluconate, $20 \mathrm{mM}$ TEA-Cl, $20 \mathrm{mM}$ BAPTA, $4 \mathrm{mM} \mathrm{MgCl}_{2}$, $4 \mathrm{mM}$ ATP, $0.4 \mathrm{mM}$ GTP, and $30 \mathrm{mM}$ HEPES (pH 7.3, adjusted with $\mathrm{CsOH})$. For recording of CF- or PF-EPSCs, picrotoxin $(100 \mu \mathrm{M}$, Tocris) was added to normal ACSF to block inhibitory synaptic transmission. For recording of mIPSCs, TTX $(1 \mu \mathrm{M}$, Wako) and NBQX $(10 \mu \mathrm{M}$, Tocris) were added to the normal ACSF. For recording of stimulus-response curves of PF-EPSCs, the pipette solution was composed of $65 \mathrm{mM} \mathrm{K}$-gluconate, $65 \mathrm{mM}$ Cs-

methanesulfonate, $10 \mathrm{mM} \mathrm{KCl,} 5 \mathrm{mM}$ sucrose, $0.4 \mathrm{mM}$ EGTA, $20 \mathrm{mM}$ HEPES, 1 $\mathrm{mM} \mathrm{MgCl}, 4 \mathrm{mM} \mathrm{ATP}$, and $1 \mathrm{mM}$ GTP (pH 7.3, adjusted with $\mathrm{CsOH}$ ).

Stimulation pipettes were filled with normal ACSF and placed on the ML. Square pulses were applied for focal stimulation (duration, $0.1 \mathrm{~ms}$; amplitude, from 0 to 90 V). To elicit mGluR1-mediated currents, PFs were stimulated five times at $100 \mathrm{~Hz}$ in the presence of NBQX $(20 \mu \mathrm{M})$ and picrotoxin $(100 \mu \mathrm{M})$. CFs were repetitively stimulated at $0.2 \mathrm{~Hz}$ by an electrode placed in the IGL around the PC soma. The stimulation pipette was systematically moved around the $\mathrm{PC}$ soma. The number of CFs innervating the recorded PC was estimated as the number of discrete CF-EPSC steps during gradually increasing stimulus intensity. Approximately half of the mice in each experimental group were examined and analyzed blinded for their genotypes or experimental operations.

Anterograde labeling of CFs. Under anesthesia with isoflurane, a glass pipette filled with a solution of Alexa Fluor 568-conjugated dextran (10\% in PBS, 10,000 MW; Thermo Fisher Scientific) was stereotaxically administered to the IO of C57BL/6 mice at P7-P9. The tracer was injected iontophoretically with pulse current $(10 \mu \mathrm{A}, 700 \mathrm{~ms})$ at $0.5 \mathrm{~Hz}$ for $20 \mathrm{~min}$. After tracer injection, the wound was stitched with silk sutures, and mice were moved to a cage with a heating lamp. After recovery from anesthesia, mice were moved to their home cage. After $2-3$ days recovery, mice were deeply anesthetized with pentobarbital $\left(100 \mathrm{mg} \mathrm{kg}^{-1}\right.$ body weight) and transcardially fixed with $4 \%$ PFA for immunohistochemical experiments. Images were taken using the same procedure described in the immunostaining section above. We omitted mice from the analysis if the Ibalpositive cells on the surface of the cerebellar cortex (Fig. 1a) were heavily stained by Alexa Fluor 568-conjugated dextran that leaked from the injection sites in the medulla. In such mice, microglia with Alexa Fluor 568 signals were very frequently observed in the cerebellum, probably because some of the stained microglia on the surface of the brain migrated into the developing cerebellum ${ }^{4}$.

In some experiments (Fig. $5 \mathrm{~g}, \mathrm{~h}), 3$ - AP $\left(75 \mu \mathrm{g} \mathrm{g}^{-1}\right.$ body weight, Sigma-Aldrich), harmaline (15 $\mu \mathrm{g} \mathrm{g}^{-1}$ body weight, Sigma-Aldrich), and nicotinamide (300 $\mathrm{g} \mathrm{g} \mathrm{g}^{-1}$ body weight, Sigma-Aldrich) were injected intraperitoneally at P9, after tracer injection at P7, to induce degeneration of CFs. Harmaline and nicotinamide were administered 3 and $4.5 \mathrm{~h}$ after 3 -AP, respectively. In these experiments, mice were fixed at P14 for histological assessment.

Intracerebellar liposome application. C57BL/6 mice at P6-P7 or P10-P11 were anesthetized with isoflurane, and the occipital bone and the dura over cerebellar lobules VI-VIII were carefully removed. A volume of $\sim 200 \mathrm{nl}$ of control or clodronate liposomes (\#160-0364-1 or \#160-0432-1, Katayama Chemical) was injected over 20 min into lobules VI, VII, or VIII of the cerebellar vermis at a depth of 200-250 $\mu \mathrm{m}$ using a glass capillary and a microinjector (NANOJECT II, Drum mond Scientific, or UMP3 ultra micro pump, WPI). After tracer injection, the wound was stitched with silk sutures, and mice were moved to a cage with a heating lamp. After recovery from anesthesia, mice were moved to their home cage with free access to food and water. Innervation by CFs in the injected lobule was examined electrophysiologically at P21-P31.

Intraperitoneal drug applications. LPS (from E. coli O111: B4; Sigma-Aldrich) was intraperitoneally injected into C57BL/6 mice once per day from P7 to P11 or P12 to P16. The doses of LPS were set to 0.3 and $1.5 \mu \mathrm{g} \mathrm{g}^{-1}$ of body weight, and the same volume of PBS was injected as a control. Innervation by CFs was examined electrophysiologically at P21-P32.

Diazepam (10 $\mathrm{g} \mathrm{g}^{-1}$ of body weight; Sigma-Aldrich) was administered to Csf1r-cKO mice once per day from P9 to P12. As a control, vehicle solution, which was composed of $40 \%$ propylene glycol, $10 \%$ ethanol, and $50 \%$ distilled water, was administered to a different age-matched group of Csflr-cKO mice. Innervation by CFs was examined electrophysiologically at P25-P41.

Statistical analysis. No statistical method was used to decide sample sizes, but they were chosen based on previous studies with similar methodologies. No data, except for Fig. 6 (the detail is explained in Anterograde labeling of CFs section in 
the Methods), were excluded from the analysis. For all pharmacological experiments, mice were randomly chosen from amongst littermates for the drug or vehicle injections. Throughout the text and figures, $n$ represents the number of PCs analyzed unless otherwise stated in the figure legends. Averaged values were represented as mean \pm SEM. In graphs for electrophysiological experiments, bar graphs representing mean values and the individual data points are displayed in parallel. In boxplots, center lines, whiskers, dots, and bonds of box represent the median, the 10th/90th, the 5th/95th, and the 25th/75th percentiles, respectively. Statistical significance was assessed by two-sided $t$ test or the Mann-Whitney $U$ test, depending on whether the data sets passed the normality test and equal variance test, unless otherwise stated in the text. Statistical analyses were conducted with SigmaPlot 12.1 (Systat Software) and $p$ values smaller than 0.001 were described as $p<0.001$, otherwise actual values were described in the text. Differences between two samples were considered statistically significant if the $p$ value was less than 0.05 .

Data availability. The authors declare that the main data supporting the findings of this study are available within the article and its Supplementary Information files. Data that support the findings of this study are available from the corresponding author upon reasonable request.

Received: 3 November 2017 Accepted: 23 May 2018

Published online: 19 July 2018

\section{References}

1. Ginhoux, F. et al. Fate mapping analysis reveals that adult microglia derive from primitive macrophages. Science 330, 841-845 (2010).

2. Ashwell, K. Microglia and cell death in the developing mouse cerebellum. Brain Res. Dev. Brain Res. 55, 219-230 (1990).

3. Boya, J., Calvo, J. L., Carbonell, A. L. \& Borregon, A. A lectin histochemistry study on the development of rat microglial cells. J. Anat. 175, 229-236 (1991).

4. Cuadros, M. A. et al. Microglia development in the quail cerebellum. J. Comp. Neurol. 389, 390-401 (1997).

5. Cunningham, C. L., Martinez-Cerdeno, V. \& Noctor, S. C. Microglia regulate the number of neural precursor cells in the developing cerebral cortex. J. Neurosci. 33, 4216-4233 (2013).

6. Dalmau, I., Finsen, B., Zimmer, J., Gonzalez, B. \& Castellano, B. Development of microglia in the postnatal rat hippocampus. Hippocampus 8, 458-474 (1998).

7. Perez-Pouchoulen, M., VanRyzin, J. W. \& McCarthy, M. M. Morphological and phagocytic profile of microglia in the developing rat cerebellum. eNeuro 2, ENEURO.0036-15.2015 (2015).

8. Squarzoni, P. et al. Microglia modulate wiring of the embryonic forebrain. Cell Rep. 8, 1271-1279 (2014).

9. Shigemoto-Mogami, Y., Hoshikawa, K., Goldman, J. E., Sekino, Y. \& Sato, K. Microglia enhance neurogenesis and oligodendrogenesis in the early postnatal subventricular zone. J. Neurosci. 34, 2231-2243 (2014).

10. Kettenmann, H., Hanisch, U. K., Noda, M. \& Verkhratsky, A. Physiology of microglia. Physiol. Rev. 91, 461-553 (2011).

11. Sierra, A., Abiega, O., Shahraz, A. \& Neumann, H. Janus-faced microglia: beneficial and detrimental consequences of microglial phagocytosis. Front. Cell. Neurosci. 7, 6 (2013).

12. Hoshiko, M., Arnoux, I., Avignone, E., Yamamoto, N. \& Audinat, E. Deficiency of the microglial receptor CX3CR1 impairs postnatal functional development of thalamocortical synapses in the barrel cortex. J. Neurosci. 32, 15106-15111 (2012).

13. Marin-Teva, J. L. et al. Microglia promote the death of developing Purkinje cells. Neuron 41, 535-547 (2004).

14. Riccomagno, M. M. \& Kolodkin, A. L. Sculpting neural circuits by axon and dendrite pruning. Annu. Rev. Cell Dev. Biol. 31, 779-805 (2015).

15. Hashimoto, K. \& Kano, M. Synapse elimination in the developing cerebellum. Cell. Mol. Life Sci. 70, 4667-4680 (2013).

16. Lim, S. H. et al. Neuronal synapse formation induced by microglia and interleukin 10. PLoS ONE 8, e81218 (2013).

17. Miyamoto, A. et al. Microglia contact induces synapse formation in developing somatosensory cortex. Nat. Commun. 7, 12540 (2016).

18. Parkhurst, C. N. et al. Microglia promote learning-dependent synapse formation through brain-derived neurotrophic factor. Cell 155, 1596-1609 (2013).

19. Bialas, A. R. \& Stevens, B. TGF- $\beta$ signaling regulates neuronal C1q expression and developmental synaptic refinement. Nat. Neurosci. 16, 1773-1782 (2013).

20. Kim H. J. et al. Deficient autophagy in microglia impairs synaptic pruning and causes social behavioral defects. Mol. Psychiatry 22, 1576-1584 (2017).

21. Paolicelli, R. C. et al. Synaptic pruning by microglia is necessary for normal brain development. Science 333, 1456-1458 (2011).
22. Schafer, D. P. et al. Microglia sculpt postnatal neural circuits in an activity and complement-dependent manner. Neuron 74, 691-705 (2012).

23. Tremblay, M. E., Lowery, R. L. \& Majewska, A. K. Microglial interactions with synapses are modulated by visual experience. PLoS Biol. 8, e1000527 (2010).

24. Hashimoto, K., Ichikawa, R., Kitamura, K., Watanabe, M. \& Kano, M. Translocation of a "winner" climbing fiber to the Purkinje cell dendrite and subsequent elimination of "losers" from the soma in developing cerebellum. Neuron 63, 106-118 (2009).

25. Kurihara, H. et al. Impaired parallel fiber->Purkinje cell synapse stabilization during cerebellar development of mutant mice lacking the glutamate receptor $\delta 2$ subunit. J. Neurosci. 17, 9613-9623 (1997).

26. Crepel, F. Regression of functional synapses in the immature mammalian cerebellum. Trends Neurosci. 5, 266-269 (1982).

27. Ichikawa, R. et al. Developmental switching of perisomatic innervation from climbing fibers to basket cell fibers in cerebellar Purkinje cells. J. Neurosci. 31, 16916-16927 (2011).

28. Hashimoto, K. et al. Roles of glutamate receptor $\delta 2$ subunit (GluR $\delta 2)$ and metabotropic glutamate receptor subtype 1 (mGluR1) in climbing fiber synapse elimination during postnatal cerebellar development. J. Neurosci. 21 9701-9712 (2001)

29. Nakayama, H. et al. GABAergic inhibition regulates developmental synapse elimination in the cerebellum. Neuron 74, 384-396 (2012).

30. Uesaka, N. et al. Retrograde semaphorin signaling regulates synapse elimination in the developing mouse brain. Science 344, 1020-1023 (2014).

31. Altman J., Bayer S. A. Development of the Cerebellar System: In Relation to it Evolution, Structure, and Functions (CRC Press, Boca Raton, Florida, 1997).

32. Ke, M. T. et al. Super-resolution mapping of neuronal circuitry with an indexoptimized clearing agent. Cell Rep. 14, 2718-2732 (2016).

33. Hashimoto, K. et al. Influence of parallel fiber-Purkinje cell synapse formation on postnatal development of climbing fiber-Purkinje cell synapses in the cerebellum. Neuroscience 162, 601-611 (2009).

34. Dai, X. M. et al. Targeted disruption of the mouse colony-stimulating factor 1 receptor gene results in osteopetrosis, mononuclear phagocyte deficiency, increased primitive progenitor cell frequencies, and reproductive defects. Blood 99, 111-120 (2002)

35. Erblich, B., Zhu, L., Etgen, A. M., Dobrenis, K. \& Pollard, J. W. Absence of colony stimulation factor-1 receptor results in loss of microglia, disrupted brain development and olfactory deficits. PLoS ONE 6, e26317 (2011).

36. Schafer, D. P., Lehrman, E. K., Heller, C. T. \& Stevens, B. An engulfment assay: a protocol to assess interactions between CNS phagocytes and neurons. J. Vis. Exp. 88, e51482 (2014).

37. Zhang, Y., Forster, C., Milner, T. A. \& Iadecola, C. Attenuation of activityinduced increases in cerebellar blood flow by lesion of the inferior olive. Am. J. Physiol. Heart Circ. Physiol. 285, H1177-H1182 (2003).

38. Llinas, R., Walton, K., Hillman, D. E. \& Sotelo, C. Inferior olive: its role in motor learing. Science 190, 1230-1231 (1975).

39. Wecker, L., Marrero-Rosado, B., Engberg, M. E., Johns, B. E. \& Philpot, R. M 3-Acetylpyridine neurotoxicity in mice. Neurotoxicology 58, 143-152 (2017).

40. Hicks, S. P. Pathologic effects of antimetabolites. I. Acute lesions in the hypothalamus, peripheral ganglia, and adrenal medulla caused by 3-acetyl pyridine and prevented by nicotinamide. Am. J. Pathol. 31, 189-199 (1955).

41. Simantov, R., Snyder, S. H. \& Oster-Granite, M. L. Harmaline-induced tremor in the rat: abolition by 3 -acetylpyridine destruction of cerebellar climbing fibers. Brain Res. 114, 144-151 (1976).

42. Ichikawa, R. et al. Territories of heterologous inputs onto Purkinje cell dendrites are segregated by mGluR1-dependent parallel fiber synapse elimination. Proc. Natl Acad. Sci. USA 113, 2282-2287 (2016).

43. Fagiolini, M. \& Hensch, T. K. Inhibitory threshold for critical-period activation in primary visual cortex. Nature 404, 183-186 (2000).

44. Elmore, M. R. et al. Colony-stimulating factor 1 receptor signaling is necessary for microglia viability, unmasking a microglia progenitor cell in the adult brain. Neuron 82, 380-397 (2014).

45. Nandi, S. et al. The CSF-1 receptor ligands IL-34 and CSF-1 exhibit distinct developmental brain expression patterns and regulate neural progenitor cell maintenance and maturation. Dev. Biol. 367, 100-113 (2012).

46. Murase, S. \& Hayashi, Y. Expression pattern and neurotrophic role of the cfms proto-oncogene M-CSF receptor in rodent Purkinje cells. J. Neurosci. 18 10481-10492 (1998)

47. Wang, Y., Berezovska, O. \& Fedoroff, S. Expression of colony stimulating factor-1 receptor (CSF-1R) by CNS neurons in mice. J. Neurosci. Res. 57, 616-632 (1999).

48. Imai, Y., Ibata, I., Ito, D., Ohsawa, K. \& Kohsaka, S. A novel gene ibal in the major histocompatibility complex class III region encoding an EF hand protein expressed in a monocytic lineage. Biochem. Biophys. Res. Commun. 224, 855-862 (1996).

49. D’Mello, C. \& Swain, M. G. Immune-to-brain communication pathways in inflammation-associated sickness and depression. Curr. Top. Behav. Neurosci. 31, 73-94 (2017). 
50. Bosman, L. W. et al. Requirement of TrkB for synapse elimination in developing cerebellar Purkinje cells. Brain Cell Biol. 35, 87-101 (2006).

51. Rico, B., Xu, B. \& Reichardt, L. F. TrkB receptor signaling is required for establishment of GABAergic synapses in the cerebellum. Nat. Neurosci. 5, 225-233 (2002).

52. Johnson, E. M., Craig, E. T. \& Yeh, H. H. TrkB is necessary for pruning at the climbing fibre-Purkinje cell synapse in the developing murine cerebellum. $J$. Physiol. 582, 629-646 (2007).

53. Choo, $M$. et al. Retrograde BDNF to TrkB signaling promotes synapse elimination in the developing cerebellum. Nat. Commun. 8, 195 (2017).

54. Serantes, R. et al. Interleukin-1beta enhances GABAA receptor cell-surface expression by a phosphatidylinositol 3-kinase/Akt pathway: relevance to sepsis-associated encephalopathy. J. Biol. Chem. 281, 14632-14643 (2006).

55. Stuck, E. D. et al. Tumor necrosis factor alpha mediates GABA(A) receptor trafficking to the plasma membrane of spinal cord neurons in vivo. Neural Plast. 2012, 261345 (2012).

56. Kawamura, Y. et al. Spike timing-dependent selective strengthening of single climbing fibre inputs to Purkinje cells during cerebellar development. Nat. Commun. 4, 2732 (2013).

57. Horn, K. P., Busch, S. A., Hawthorne, A. L., van Rooijen, N. \& Silver, J. Another barrier to regeneration in the CNS: activated macrophages induce extensive retraction of dystrophic axons through direct physical interactions. J. Neurosci. 28, 9330-9341 (2008).

58. Kitayama, M., Ueno, M., Itakura, T. \& Yamashita, T. Activated microglia inhibit axonal growth through RGMa. PLoS ONE 6, e25234 (2011).

59. Li, J., Chen, K., Zhu, L., Pollard, J. W. Conditional deletion of the colony stimulating factor-1 receptor (c-fms proto-oncogene) in mice. Genesis $\mathbf{4 4}$, 328-335 (2011).

\section{Acknowledgements}

We thank M. Mitsushima, M. Nonaka, M. Takata, R. Takimoto, H. Teramichi, K. Yamaoka, S. Suzuki, and R. Kubo for their assistance in preliminary experiments, and M. Kawamura for technical assistance. This work was supported by Grants-in Aid for Scientific Research (15K183420A and 17K070580A to H.N., 26350979 to M.A., $17 \mathrm{H} 01387$ to S.O., $25000015,17 \mathrm{H} 03551$, and $18 \mathrm{H} 04947$ to K.H., 25117006 to S.O. and K.H.) from the Ministry of Education, Culture, Sports, Science and Technology of Japan. This work was also supported by the Strategic Research Program for
Brain Sciences (17dm0107093h0002) from AMED to K.H. We thank Ann Turnley, Ph.D., from Edanz Group (www.edanzediting.com/ac) for editing a draft of this manuscript.

\section{Author contributions}

H.N. and K.H. designed the study. H.N., M.A., C.M., and T.I. performed experiments. H N., M.A., C.M., T.I., and K.H. analyzed the data. M.A., T.I., S.O., and K.S. contributed reagents/analysis tools. H.N., M.A., C.M., T.I. S.O., K.S., and K.H. wrote the paper.

\section{Additional information}

Supplementary Information accompanies this paper at https://doi.org/10.1038/s41467 018-05100-z.

Competing interests: The authors declare no competing interests.

Reprints and permission information is available online at http://npg.nature.com/ reprintsandpermissions/

Publisher's note: Springer Nature remains neutral with regard to jurisdictional claims in published maps and institutional affiliations.

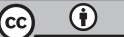

Open Access This article is licensed under a Creative Commons Attribution 4.0 International License, which permits use, sharing, adaptation, distribution and reproduction in any medium or format, as long as you give appropriate credit to the original author(s) and the source, provide a link to the Creative Commons license, and indicate if changes were made. The images or other third party material in this article are included in the article's Creative Commons license, unless indicated otherwise in a credit line to the material. If material is not included in the article's Creative Commons license and your intended use is not permitted by statutory regulation or exceeds the permitted use, you will need to obtain permission directly from the copyright holder. To view a copy of this license, visit http://creativecommons.org/ licenses/by/4.0/.

(c) The Author(s) 2018 